Hrvoje Kozmar

Davide Allori

Gianni Bartoli

Claudio Borri

https://doi.org/10.21278/TOF.43302

ISSN 1333-1124

eISSN 1849-1391

\title{
WIND CHARACTERISTICS IN THE WAKE OF A NON-ROTATING WIND TURBINE CLOSE TO A HILL
}

\begin{abstract}
Summary
Hilly terrain may considerably influence energy yield and structural loading of wind turbines. Wind energy developers are therefore required to accurately predict wind characteristics at the wind farm site already in the designing phase. It is necessary to determine how particular hill shape and size affect wind characteristics at the location of wind turbines operating in the wake of a hill. In order to address this complex issue, small-scale laboratory experiments were carried out in a boundary layer wind tunnel (BLWT). The focus was on characteristics of the flow at the same position downstream of the hill and the wind turbine models studied on various sizes and shapes of the hill model. Three hill models (a small and a large hill with laterally uniform heights and a hill with a bay) were exposed to the thermally neutrally stratified atmospheric boundary layer (ABL) simulation. In each experiment, there was only one hill model in the BLWT test section. The configuration without the hill, i.e. the flat terrain model, was tested as a reference case. Downstream of the hill model, there was a single (alone-standing) wind turbine model. Flow characteristics were studied downstream (behind) the wind turbine model. The wind turbine model was in the parking position in order to study wind characteristics in the situation with a strong wind when there is no rotation of rotor blades. The experimental results indicate a velocity decrease and stronger turbulence in the wind turbine wake in the presence of a hill, which trend is more pronounced in the case of larger hills. Strong velocity gradients are observed around the hub height in the large hill configuration, while this velocity deficit is the smallest in the hill with a bay configuration due to flow channeling. The velocity power spectra reveal the characteristic peak due to the vortex shedding from the hill ridge. The obtained results provide a new insight into the turbulent wake characteristics of a single wind turbine placed downstream of a hill with a potential to serve as a validation tool for computational studies.
\end{abstract}

Key words: $\quad$ wind energy, wind turbine, hilly terrain, aerodynamic characteristics, wind tunnel experiments.

\section{Introduction}

The ability of wind turbines built in the orographically complex, hilly terrain to extract kinetic energy from the flow decreases due to wind characteristics significantly different from the 'classical' atmospheric boundary layer (ABL) flow developing above a flat terrain that 
wind turbines are originally designed for. In addition, unsteady wind loading of wind energy structures in those areas can likely reveal additional, unknown features. While there is a lot of information on wind turbine aerodynamics relating to the flat terrain type, further work is still required with respect to wind characteristics in the hilly terrain that is essential if the lifetime of wind turbines is to be extended and energy production maximized.

This project builds upon the findings of previous relevant studies on wind turbine aerodynamics exposed to the ABL flow developing above a flat terrain. In particular, an overview and analysis of different wake modelling methods is reported in Crespo et al. [1], while the wake interacting with the free stream wind and the local terrain influences the total energy resource at a particular wind farm site, Whale et al. [2]. As the turbulence in the wake may affect the fatigue loading of downwind turbines within the wind farm, e.g. Kozmar et al. [3], this issue needs to be carefully investigated when designing a wind farm layout.

The wind turbine wake is commonly divided into a near and a far wake, Vermeer et al. [4]. The near wake is the region from the wind turbine to approximately one rotor diameter downwind of the turbine. It is characterized by a number of rotor blades and their aerodynamics. In this range, helical tip vortices develop due to the rotating blades and considerably determine the behaviour of the turbulent wake flow structures behind a wind turbine. In particular, Zhang et al. [5] identified localized regions of strong vorticity and swirling strength, while Hu et al. [6] characterized the dynamic wind loads and evolution of the unsteady vortex and turbulent flow structures. Chamorro and Porté-Agel [7] found an axisymmetric shape of the velocity deficit, where the turbulence intensity distribution is nonaxisymmetric and the velocity power spectra show a signature of blade tip vortices. The tip vortices are also a source of noise and blade vibration, Massouh and Dobrev [8].

The far wake is the region beyond the near wake, where the rotor design and performance become less important and wake interference with the surrounding terrain and structures starts to dominate the wake physics. An extensive knowledge on turbulent vortex flows in the wind turbine far wake is crucial for the optimal designing of wind turbines in order to improve energy yield and reduce structural fatigue of the wind turbines placed downwind within a farm as those wind turbines are directly exposed to turbulent wakes of the wind turbines placed upstream.

Small-scale experiments have commonly served as a valuable tool in studying wind turbine wakes, e.g. Alfredsson and Dahlberg [9], Ebert and Wood [10], Grant and Parkin [11], Whale et al. [12], Simms et al. [13], Vermeer [14], Medici and Alfredsson [15], Snel et al. [16], Dobrev et al. [17], Sørensen [18]. However, most previous laboratory studies were carried out assuming uniform wind conditions, e.g. Vermeer et al. [4], which are not fully representative of the highly turbulent atmospheric airflows that wind turbines are commonly subjected to. Hence, some recent wind tunnel studies were carried out to further elucidate the effects of the ABL on the wake characteristics, e.g. Chamorro and Porté-Agel [7], [19], [20], Markfort et al. [21], Zhang et al. [22].

Hancock and Pascheke [23] investigated wake characteristics influenced by the thermal stratification, while Cal et al. [24] indicated that the fluxes of kinetic energy associated with the Reynolds shear stress are of the same order of magnitude as the power extracted by wind turbines thus indicating the importance of vertical transport in the boundary layer; these issues are expected to additionally gain on complexity in the marine wind environment, e.g. Hadžić et al. [25].

While previous studies highlight the necessity of considering the effects of boundary layer flows on the wind turbine wake, up-to-date the studies on effects of strong topographical 
disturbance (for example a hill) on wind turbine wake characteristics are quite rare, whereas such strong topographical features are likely to substantially influence the unsteady wake physics and wind turbine loads, e.g. Botta et al. [26]. Hence, it is of great interest to learn more about the wind turbine aerodynamic characteristics in a hilly terrain. This will result in an effective design of wind turbines in those areas with respect to the energy yield, structural loading and fatigue.

There are generally two important issues when designing wind turbines in complex terrain, i.e. the first one is the flow development downstream of a hill to determine a distance where the turbulent atmospheric flow assumes the original undisturbed characteristics comparable to those upstream of a hill. At this distance, wind turbines will experience similar wind loads and they will yield the amount of energy comparable to that generated by the wind turbines situated upstream of a hill. This issue was previously analyzed in Kozmar et al. [27], where the results of a development of the mean velocity and turbulence intensity in the wake of different hill types were provided in the form of design curves. Also, this information may be important for design purposes in order to allow for calculating projected structural loads and wind energy yield in the hill far wake.

Another important issue, which is the focus of the present study, are flow characteristics at a fixed position downstream of the hill and the wind turbine studied on various sizes and shapes of the hill. It is particularly important to address this issue because there are many regions around the world where wind turbines are placed immediately downstream of a hill, i.e. there is no enough flat terrain downstream of a hill to allow for the flow to recover from a disturbance caused by the hill and due to that fact the wind turbines are designed in the hill near wake. In this case, it is required to exactly know the wind characteristics at the fixed position in the hill wake caused by the hills of various sizes and shapes as various hill types yield substantially different flow characteristics at the same position in the hill wake.

\section{Experimental setup}

The experiments were carried out in the CRIACIV boundary layer wind tunnel, which is described in Augusti et al. [28]. The standard wind tunnel procedures (Simiu and Scanlan [29], Dyrbye and Hansen [30], Holmes [31], Mattuella et al. [32]) were adopted for this purpose. The experimental setup was described in Kozmar et al. [27]; however, for the sake of the completeness of the present study, it is also provided here in a modified form.

This wind tunnel is designed as an open-return (Eiffel) suction-type wind tunnel with a closed test section. Flow velocity through the test section can be regulated between $0 \mathrm{~m} / \mathrm{s}$ and $30 \mathrm{~m} / \mathrm{s}$ by both adjusting the pitch blade angle and regulating the speed of the fan powered by a $160 \mathrm{~kW}$ engine, where the fan is placed at the outlet of the test section. The total length of the wind tunnel test section is approximately $22 \mathrm{~m}$. The test section is $1.6 \mathrm{~m}$ high and $2.2 \mathrm{~m}$ wide at the outlet of the nozzle, i.e. at the inlet of the test section. Due to diverging sidewalls in the longitudinal direction whose purpose is to avoid pressure gradients, the test section width at the centre of the turntable is $2.4 \mathrm{~m}$.

In this wind tunnel, it is possible to simulate the ABL flow and turbulence along the first $11 \mathrm{~m}$ of the test section ( $8 \mathrm{~m}$ long fetch and $3 \mathrm{~m}$ at the turntable). At the turntable, the horizontal flow incidence angle on structural models can be varied. The ABL simulation upstream of the hill model was created by applying the Counihan [33] technique. The flow conditioning devices encompass three Counihan $1.53 \mathrm{~m}$ high quarter-elliptic constant-wedgeangle spires, a castellated barrier wall and a fetch of surface roughness elements, Fig. 1. 
a)

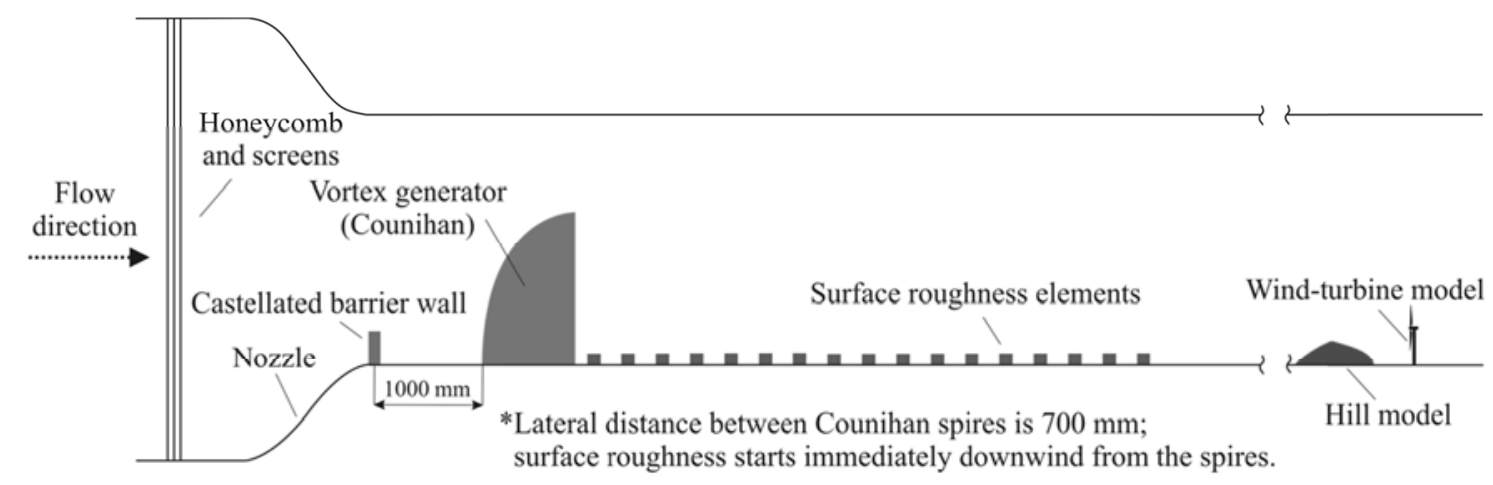

b)

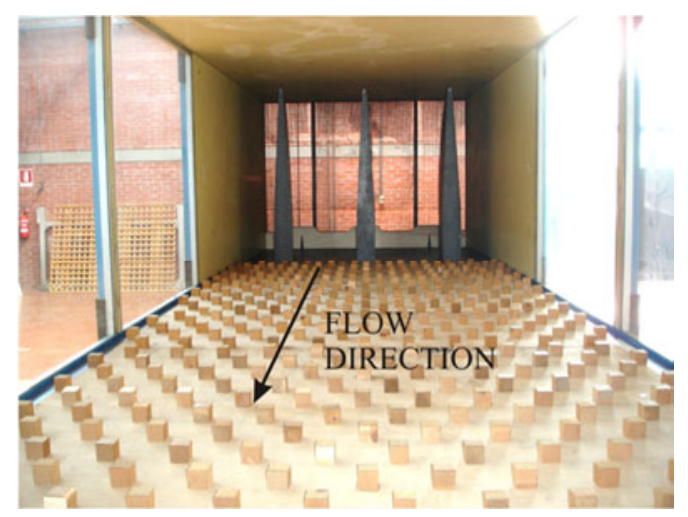

Fig. 1 Experimental setup for the wind tunnel ABL simulation including the castellated barrier wall, three Counihan vortex generators and surface roughness elements: a) schematic side view, b) photograph

The castellated barrier wall used in this study is presented in Fig. 2, while the surface roughness consisted of $0.05 \mathrm{~m}$ high, 0.05 wide and $0.05 \mathrm{~m}$ long wooden cubes placed in a staggered pattern with a lateral distance between the cubes of $0.145 \mathrm{~m}$ in the lateral and 0.110 $\mathrm{m}$ in the longitudinal direction.

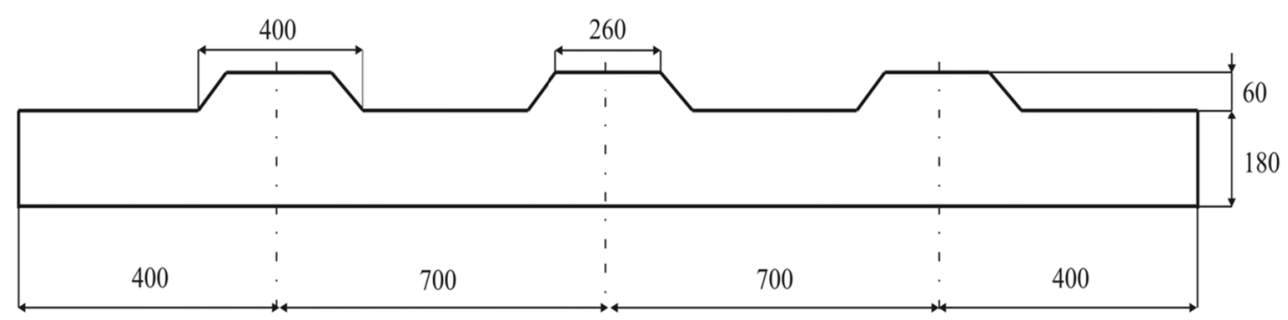

Fig. 2 Castellated barrier wall used in this study (all measures in $\mathrm{mm}$ )

Three different hill models were used as well as a smooth surface representing a flat terrain (FT), Fig. 3. The hill models were designed to closely represent realistic terrain conditions that commonly appear in the full-scale, e.g. Lepri et al. [34], [35], rather than some generic shape. 
a) Flat terrain (FT)

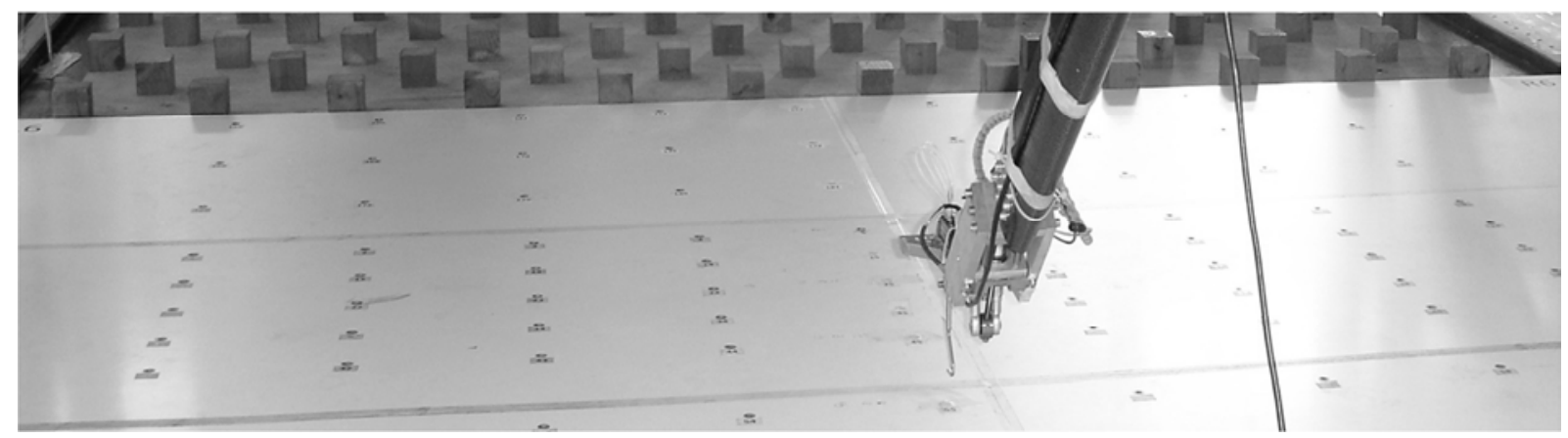

b) Small hill (SH) and large hill (LH)

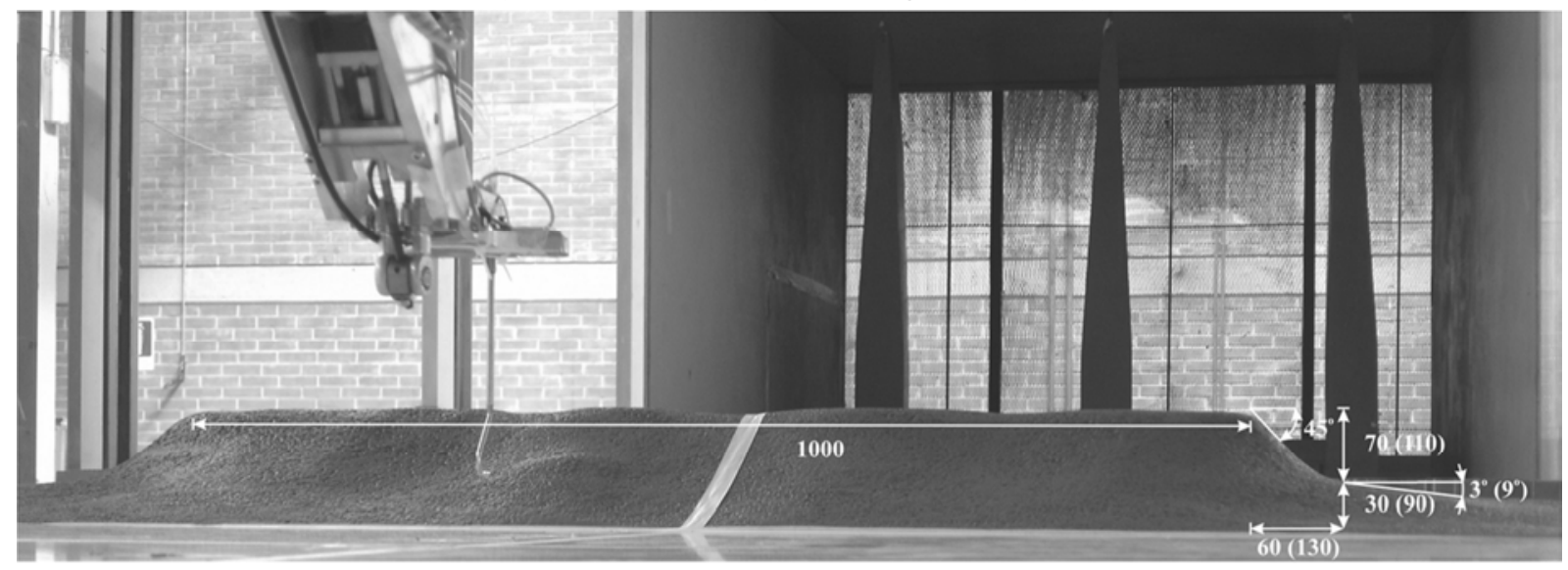

c) Hill with a bay (BAY)

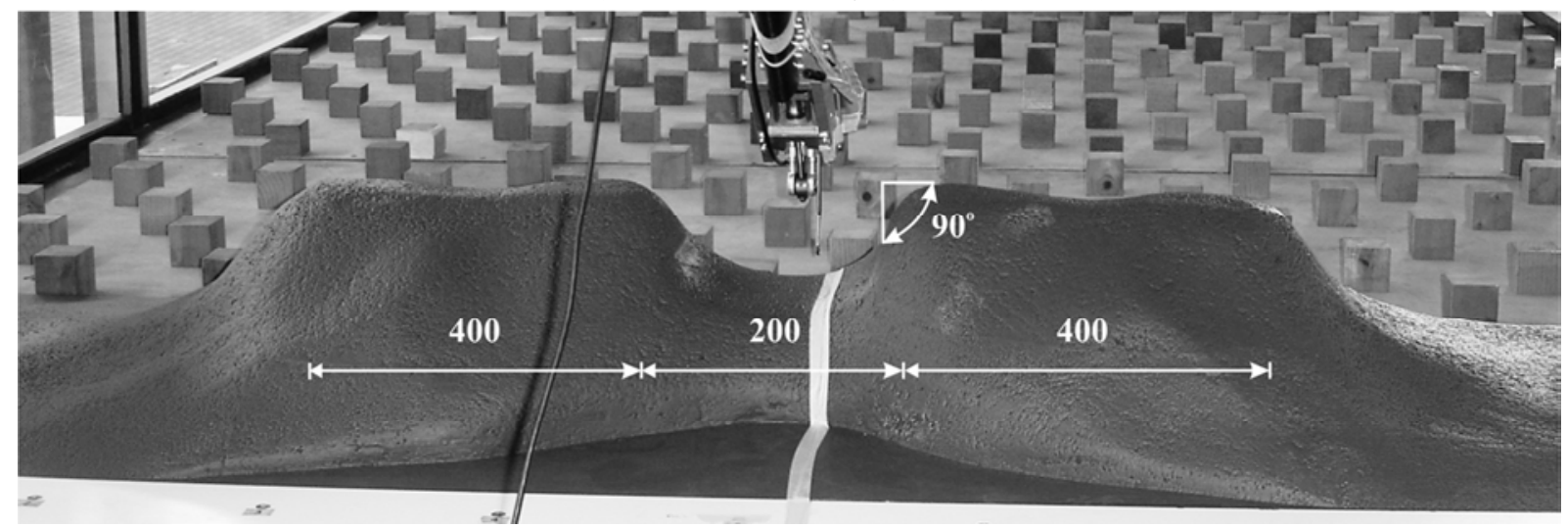

Fig. 3 Measures of the hill models (lengths in mm): a) flat terrain (FT); b) small hill (SH) and large hill (LH), where SH measures are provided first, LH measures are provided in brackets, while lateral length $(1000 \mathrm{~mm})$ and lateral slope $\left(45^{\circ}\right)$ are the same for SH and LH; the slopes (both windward and leeward) are $18^{\circ}$ for $\mathrm{SH}$ and $34^{\circ}$ for LH; c) hill with a bay (BAY), where the measures not indicated are the same as for LH

Each hill model is $600 \mathrm{~mm}$ long in the main flow direction (along the wind tunnel test section) and $1000 \mathrm{~mm}$ wide (lateral to the main flow direction). The small hill ( $\mathrm{SH}$ ) model is $100 \mathrm{~mm}$ uniformly high, the large hill (LH) model is $200 \mathrm{~mm}$ uniformly high, while the hill with a bay (BAY) is $200 \mathrm{~mm}$ high, whereas the height decreases to $100 \mathrm{~mm}$ height in the lateral centre of the hill.

The geometry of the hill models was selected to achieve the wind tunnel blockage below the commonly accepted critical value of 5\%, e.g. Simiu and Scanlan [29]. At the same 
time, $\mathrm{LH}$ twice as high as $\mathrm{SH}$, and BAY having a larger height equal to $\mathrm{LH}$ and the smaller height equal to SH allow for resolving the dominant flow phenomena due to the height and the shape of the hill model. The surface of the hill models is quite smooth with a very low surface roughness, while the minor irregularities in the hilly terrains, which were manufactured in order to make the hill models look more realistic, were not expected to have any appreciable influence on the flow in the wake of the hill models.

The leading edge of all terrain types was placed $5.3 \mathrm{~m}(3.5 \delta)$ downstream of the vortex generators' trailing edge thus allowing for the ABL simulation to fully develop along the fetch prior to reaching the hill model. Here, $\delta$ is the vortex generators' height and the approximate thickness of the ABL simulation. This is in agreement with the original Counihan [33] method, where it was indicated that the ABL simulation became fully developed and uniform three to four ABL simulation heights $\delta$ downstream of the trailing edge of the vortex generators. Downstream of the trailing edge of the hill, the test section surface was smooth representing flat terrain.

The wind turbine model was placed $50 \mathrm{~mm}$ downstream of the trailing edge of the hill model in the lateral centre of the hill model and the wind tunnel test section. The wind turbine model was placed close to the hill model to allow for a clear resolving of the hill effects on the flow characteristics because at this position the effects of the hills are substantially stronger than further downstream.

The cylindrical wind turbine tower with a diameter of $12 \mathrm{~mm}$ is $240 \mathrm{~mm}$ high. The diameter of the $28 \mathrm{~mm}$ long cylindrical nacelle is $16 \mathrm{~mm}$. Three $160 \mathrm{~mm}$ long turbine blades designed similar to DOWEC-NREL 5MW and EU 56.1400-2 blades were fixed in a parking position (no rotation). Non-rotating case comparable to strong wind situations (wind velocity higher than $25 \mathrm{~m} / \mathrm{s}$ ) is important, particularly for structural engineers, as this configuration needs to be carefully investigated with respect to dynamic loading and response of wind energy structures. Nevertheless, the effects of a complex terrain on the wind turbine wake would need to be further addressed for rotating blades as well as previous studies, e.g. Zhang et al. [5], Hu et al. [6], Whale et al. [12], indicated a considerable influence of rotating blades on highly turbulent and three-dimensional wake flow. The adopted rotor blade length to wind turbine hub height ratio corresponds to a common wind turbine design, e.g. Siemens 6.0 MW Sapiens. The ratios between the wind turbine hub height and the height of different hills are reported in Table 1.

Table 1 Ratios of the height of different hills to the wind turbine hub height. *The hill with a bay model is $200 \mathrm{~mm}$ high with a slope to the $100 \mathrm{~mm}$ height in the lateral centre of the hill. Hence, there are two different ratios of the hill height to the wind turbine hub height; 0.833 at the lateral edges of the hill, 0.417 in the lateral centre of the hill

\begin{tabular}{ccccc}
\cline { 2 - 5 } & FT & SH & LH & BAY \\
\hline Hill height/Hub height & 0 & 0.417 & 0.833 & $0.833 ; 0.417^{*}$ \\
\hline
\end{tabular}

In all experiments, the blockage of the wind tunnel test section was below 5\%. As an example, one arrangement of the castellated barrier wall, vortex generators, surface roughness elements along with the hill and the wind turbine models is presented in Fig. 4. 
a)

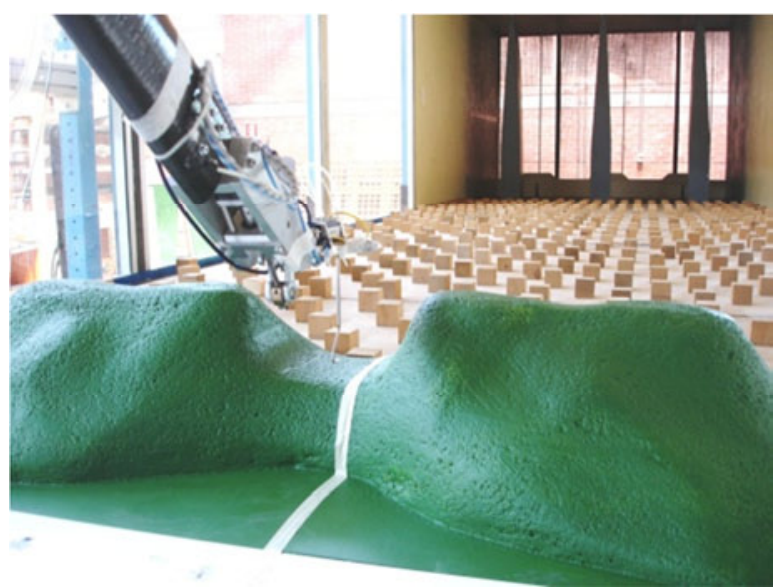

b)

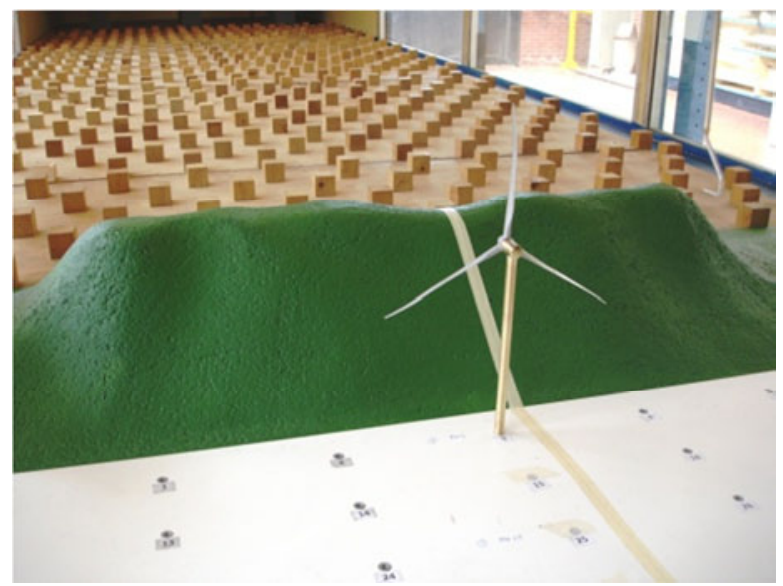

Fig. 4 Experimental setup in the wind tunnel test section: a) castellated barrier wall, vortex generators, surface roughness and the hill with a bay model, b) wind turbine model placed $50 \mathrm{~mm}$ downstream of the trailing edge of the large hill model

The measurement technique includes a conventional Aeroprobe Cobra sensor and a Prandtl-Pitot tube for velocity measurements and pressure and temperature sensors. The Cobra sensor is a conventional one with five holes and a maximum sampling frequency of 50 Hz. It is calibrated within the calibration cone of $60^{\circ}$ and generally not that suitable for measurements in the reversed flow that can likely occur downstream of the hill models. Hence, the percentage of data recorded inside the calibration cone with respect to the total number of data samples was estimated for each measuring configuration using the standard Aeroprobe software.

In the case of FT and SH the flow is not reversed, as some $99 \%$ of data samples were recorded inside the calibration cone in all of those configurations. Immediately downstream of the BAY model at the position of the wind turbine model and in the near wake at one rotor diameter $D$ downstream of the wind turbine tower $(x / D=1)$ and close to the ground surface up to $0.5 z_{\text {hub }}$, there are some indications of a reversed flow even if at least $90 \%$ of data samples were recorded inside the calibration cone in those configurations. In other BAY configurations, the number of data samples inside the calibration cone is $98 \%$ and more. The strongest reversal of the flow was observed in the case of the LH model, particularly immediately downstream of the hill model (wind turbine position and $x / D=1$ ) and close to the ground up to $0.85 z_{\text {hub }}$. In this range, the percentage of data samples recorded inside the calibration cone was between $70 \%$ and $80 \%$, while in the remaining LH configurations it was $97 \%$ and more.

In the streamlined, smooth flow, the measurement uncertainty for both the Prandtl-Pitot tube and the Aeroprobe Cobra sensor is about 1\%. This analysis indicates that the obtained data can be well adopted with respect to the studied physical phenomena, while it would be beneficial to further confirm the recordings in the immediate wake of the LH and BAY models close to the ground surface by using some experimental technique even more appropriate for measurements in the reversed flow, e.g. Particle-Image-Velocimetry (PIV).

The flow and the turbulence were analyzed with respect to the mean velocity, turbulence intensity, Reynolds stress, integral length scales of turbulence, and power spectral density of velocity fluctuations, as this set of $\mathrm{ABL}$ parameters allows for a comprehensive analysis of ABL characteristics, e.g. [36], [37], [38], [39], [40]. The ABL velocity profiles without the hill model were normalized using the reference velocity $\bar{u}_{\text {ref }}=10.2 \mathrm{~m} / \mathrm{s}$ at the reference height $z_{\text {ref }}=0.216 \mathrm{~m}$. The $z_{\text {ref }}=0.216 \mathrm{~m}$ is selected as one of the measuring points 
in the lower ABL (above the displacement height), as this way of normalization is common when studying ABL wind tunnel simulations. The wind profiles downstream of the hill were evaluated using the free stream mean velocity $\bar{u}_{\infty}=15 \mathrm{~m} / \mathrm{s}$ measured in the undisturbed free stream flow at the height of $930 \mathrm{~mm}$ and $1000 \mathrm{~mm}$ upstream of the leading edge of the hill in order to highlight the velocity reduction behind the hill as compared to the free stream flow. This analysis can be particularly interesting when making projections about the wind energy yield.

The ABL profiles were created based on the measurements obtained at 15 different heights (in vertical direction only), i.e. at the heights of 75, 90, 107, 128, 152, 182, 216, 258, $308,366,437,521,621,741,884 \mathrm{~mm}$. The near-surface velocity measurements at heights lower than $75 \mathrm{~mm}$ were not taken as they do not considerably change the shape of the investigated profiles, while the focus of this study was on the entire height range between the ground surface and the top tip height of the wind turbine. Downstream of the hill and the flat terrain models, the flow characteristics were determined based on the velocity measurements made both in the vertical and the lateral direction. In particular, the vertical profiles were made out of the measurement results in ten points, while the lateral profiles were built based on the results in five points. For both profile types, the spacing between the neighbouring measuring points was $z_{\text {hub }} / 6$, where $z_{\text {hub }}$ is the wind turbine hub height. For the vertical profile, the measuring points extend from the ground surface up to the top tip height, while for the lateral profile they were placed from the hub laterally toward the rotor radius, as reported in Fig. 5. The preliminary tests carried out with a larger number of more densely spaced measurement points indicated that the finally selected and further applied raster of points provided a satisfactory resolution for creating the profiles necessary for the analyses.

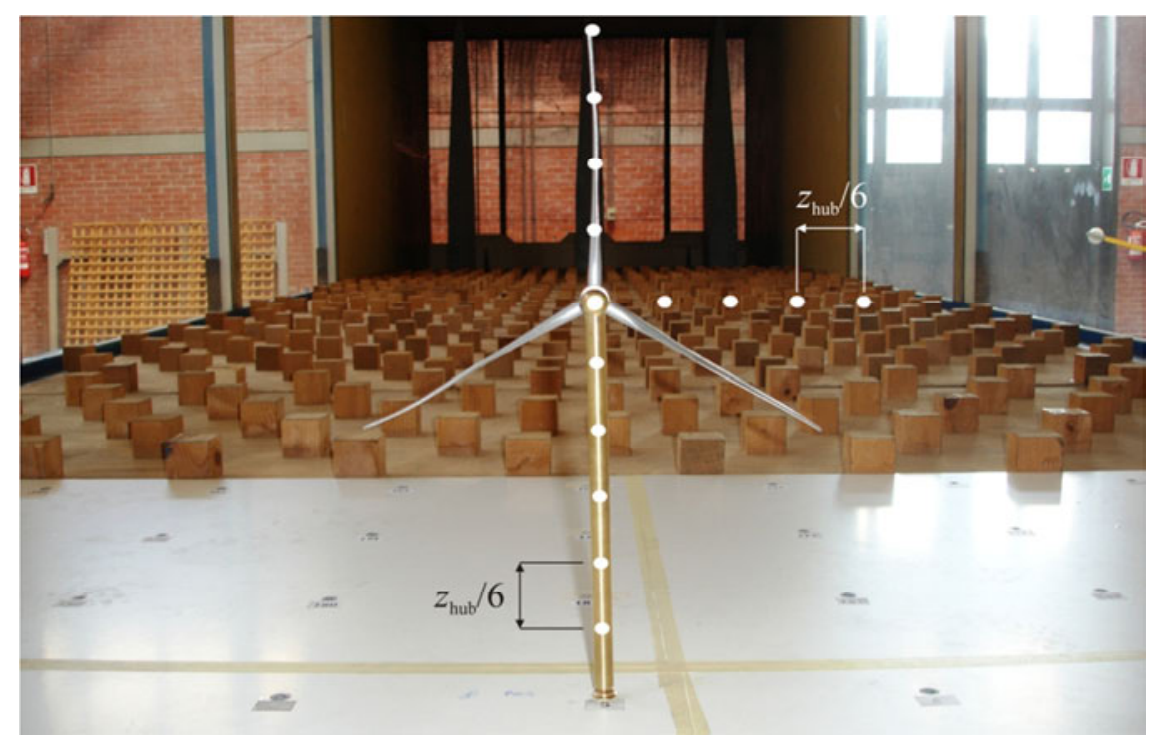

Fig. 5 Positions of the measuring points; vertical profiles made out of measurement results in ten points; lateral profiles built based on results in five points; measuring point at the hub height was used for both profiles

The initial velocity measurements were carried out to determine characteristics of the ABL simulation as well as to investigate the effects of the time record length on the ABL simulation. Hence, these initial tests were performed in the time record durations of $30 \mathrm{~s}, 60 \mathrm{~s}$, $90 \mathrm{~s}$, and $120 \mathrm{~s}$. As the obtained results differed insignificantly for the time records longer than $60 \mathrm{~s}$, the results of the ABL simulation are reported for the time record length of $120 \mathrm{~s}$, while further experiments when analyzing the wind turbine wake characteristics were carried out and reported for the time record length of $60 \mathrm{~s}$. The sampling rate was $500 \mathrm{~Hz}$ in all tests. 


\section{Results and discussion}

\subsection{Wind turbine wake characteristics}

The results of the created ABL simulation upstream of a hill model in the undisturbed flow were previously reported in [27]; however, for brevity, the important details are provided here as well as in the Appendix to complement the results for the flow characteristics at the fixed position downstream of the hill and the wind turbine studied on various sizes and shapes of the hill, which is the focus of the present study.

The ABL simulation length scale factor (300) was calculated as suggested by Cook [41] using the aerodynamic surface roughness length $z_{0}$ and the longitudinal integral length scale of turbulence $L_{u, x}$. In the wind tunnel test section without a hill model in place, the following parameters of the ABL simulation were estimated using the fitting procedure: aerodynamic surface roughness length $z_{0}$ is $6 \mathrm{~mm}$, displacement height $d$ is $50 \mathrm{~mm}$, power law exponent for the mean velocity $u$ profile in the main ( $x$ ) flow direction $\alpha$ is 0.26 , friction velocity $u_{\tau}$ is 1.2 $\mathrm{m} / \mathrm{s}$, all indicating an urban-type ABL simulation with the Prandtl constant-flux layer clearly exhibited in the lower part of the ABL simulation. The experiments were performed assuming a neutral thermal stratification of the ABL. The roughness Reynolds number $\operatorname{Re}_{\mathrm{R}}=u_{\tau} \cdot z_{0} / v$ is 476 ( $v$ is air viscosity), which is larger than 5 recommended as the minimum value for ABL wind tunnel simulations, Plate [42].

The results indicate that the created ABL simulation upstream of the hill model corresponds well to the full-scale ABL developing above the flat urban-type terrain. This ABL simulation developing above a flat terrain impinged the hill model and contributed to creating altered flow and turbulence characteristics downstream of the hill model. In particular, the power law exponent $\alpha=0.26$ with displacement height $d=15 \mathrm{~m}$ (full-scale dimensions scaled-up from the wind tunnel to full-scale using the adopted 1:300 simulation length scale) along with the aerodynamic surface roughness length $z_{0}=1.8 \mathrm{~m}$ (full-scale dimensions scaled-up using the adopted 1:300 simulation length scale) represent urban areas or very hilly or hilly areas well, e.g. Dyrbye and Hansen [30]. The characteristic full-scale measures of the hills and the wind turbine calculated by using the adopted 1:300 simulation length scale are reported in Tables 2 and 3, respectively.

Table 2 Full-scale heights of the hills calculated by using the adopted 1:300 simulation length scale. The hill with a bay model is $60 \mathrm{~m}$ high with a slope to $30 \mathrm{~m}$ in height in the lateral centre of the hill

\begin{tabular}{ccccc}
\cline { 2 - 5 } & FT & SH & LH & BAY \\
\hline Height $(z$-direction), $m$ & 0 & 30 & 60 & $60 ; 30$ \\
\hline
\end{tabular}

Table 3 Full-scale lengths of the wind turbine calculated by using the adopted 1:300 simulation length scale

\begin{tabular}{cccccc}
\cline { 2 - 6 } & $\begin{array}{c}\text { Tower } \\
\text { diameter }\end{array}$ & $\begin{array}{c}\text { Hub } \\
\text { height }\end{array}$ & $\begin{array}{c}\text { Nacelle } \\
\text { diameter }\end{array}$ & $\begin{array}{c}\text { Nacelle } \\
\text { length }\end{array}$ & $\begin{array}{c}\text { Blade } \\
\text { length }\end{array}$ \\
\hline Full-scale lengths, $\mathrm{m}$ & 3.6 & 72 & 4.8 & 8.4 & 48 \\
\hline
\end{tabular}

In addition, in the case of the flat terrain and all the studied hills, the full-scale length in the longitudinal $(x)$ direction is $180 \mathrm{~m}$, while the hills are $300 \mathrm{~m}$ wide in the lateral $(y)$ direction.

Vertical and lateral profiles of the mean flow velocity at the position of the wind turbine (without the wind turbine in place) as well as at different streamwise positions downstream of 
the wind turbine (the wind turbine in place) are presented in Figs. 6 and 7, respectively, for three different shapes of the hill, as well as for the flat terrain configuration (without the hill). While the aerodynamic drag force acting on the wind turbine is proportional to the square of the velocity, the wind turbine energy yield is proportional to the cube of the velocity. Higher velocity indicates stronger aerodynamic loads acting on the wind turbine as well as more wind energy harvested by the wind turbine, e.g. Hu et al. [6].

While from the point of view of structural loading it is both interesting to learn about the shape of the profiles and the velocity value, the mean flow velocity at the hub height has been commonly used to calculate the wind energy yield, e.g. Manwell et al. [43]. The mean velocities recorded at the hub height are therefore additionally tabulated in Fig. 6e, as those velocity deficits with respect to the undisturbed free stream flow can be particularly useful when calculating the projected wind energy yield.

a) Wind turbine position

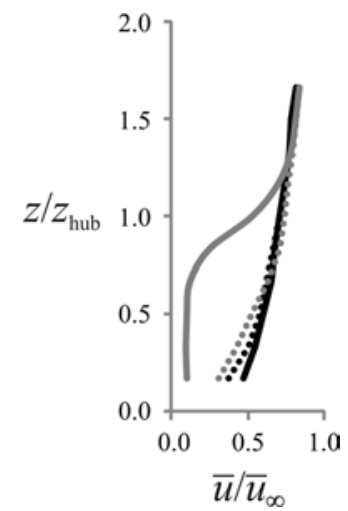

b) Wake at $x / D=1$

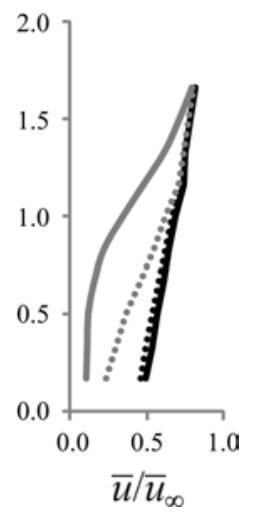

c) Wake at $x / D=3$

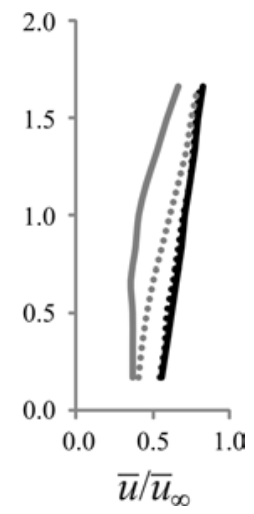

d) Wake at $x / D=5$

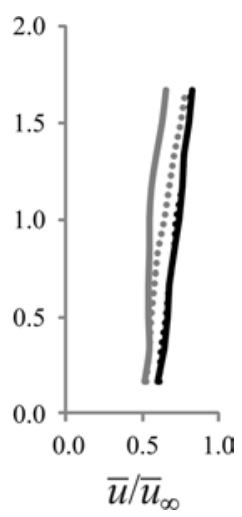

e) Normalized mean flow velocity at the hub height $\left(\bar{u}_{\text {hub }} / \bar{u}_{\infty}\right)$

\begin{tabular}{ccccc}
\cline { 2 - 5 } & FT & SH & LH & BAY \\
\hline Wind turbine position & 0.71 & 0.71 & 0.53 & 0.74 \\
Wake at $x / D=1$ & 0.69 & 0.66 & 0.34 & 0.63 \\
Wake at $x / D=3$ & 0.71 & 0.70 & 0.41 & 0.61 \\
Wake at $x / D=5$ & 0.74 & 0.72 & 0.55 & 0.64 \\
\hline
\end{tabular}

Fig. 6 a) - d) Vertical $(z)$ profiles of the longitudinal $(u)$ flow velocity at the position of the wind turbine (without wind turbine in place), as well as at different streamwise positions downstream of the wind turbine (wind turbine in place) for different terrain types: black solid line is flat terrain (without a hill), black dotted line is small hill, solid grey line is large hill, dotted grey line is hill with a bay; e) mean flow velocity at the hub height normalized using the free stream velocity; $x / D$ is nondimensionalized downstream distance of the wind turbine, $D$ is the wind turbine rotor diameter, $\bar{u}_{\infty}, \bar{u}_{\text {hub }}$ and $z_{\text {hub }}$ are mean flow velocity in the undisturbed free stream flow, mean flow velocity at the hub height and wind turbine hub height, respectively.

There are several important issues to be noted with respect to the vertical profiles of the mean velocity and velocity deficits as compared to the undisturbed, free stream flow. The velocity profiles are considerably altered by the presence of the complex orography as they are very different from the ABL velocity profile upstream of the hill, which is particularly pronounced in the case of the large hill. In particular, the large hill shadows the wind turbine to the largest extent as exhibited through the largest velocity deficit with respect to other terrains. This shadowing effect weakens further downstream of the hill, as the differences in the velocities observed for different terrains are small in the far wake at $x / D=5$. 
At the wind turbine position immediately downstream of the hill, in the case of the large hill the range of rather small velocities extends from the ground surface up to $70 \%$ of the hub height, when the velocities start to recover and reach the values observed for other terrains at $1.2 z_{\text {hub }}$. While the mean velocity profiles are rather smooth along the height for all profiles further downstream, i.e. at $x / D=3$ and 5, strong velocity gradients are observed around the hub height for the large hill when the wind turbine is placed immediately behind the hill, i.e. at the wind turbine position at $x / D=1$. While rather small velocities along the lower part of the tower of the wind turbine placed immediately downstream of the large hill at first may seem favourable with respect to the wind loading, this scenario may alter bending moments due to strong winds acting on the higher part of the wind turbine only. In addition, due to considerably different velocities in the lower and higher parts of the tower, the vortex shedding phenomena largely determining the unsteady wind loading of the wind turbine tower may exhibit significantly different features for different parts of the structure resulting in a deteriorated overall structural stability.

In the configuration without the hill, slight differences can be observed among velocity profiles recorded at various downstream distances. This is due to a roughness change between the rough floor with roughness elements (where the ABL simulation is developed) and the smooth surface without roughness elements representing the flat terrain. With respect to the velocity at the hub height, a considerable deficit is observed for all terrain types in comparison to the free stream velocity indicating a reduced wind energy yield in the presence of the hill. Immediately downstream of the leading edge of the various hills and the flat terrain, the velocity deficit is approximately $30 \%$ for the flat terrain, the small hill, and the hill with a bay, while it is as large as $47 \%$ for the large hill. For each terrain type this already considerable velocity deficit is enhanced immediately behind the wind turbine at $x / D=1$, as the wake flow is additionally retarded by the presence of the wind turbine nacelle.

Further downstream the flow recovers in the case of the flat terrain and the small hill with velocities observed at $x / D=5$ slightly exceeding the values recorded immediately downstream of the hill and remaining within the $\pm 4 \%$ margin within all distances investigated behind the hill. While in the case of the large hill the velocity at $x / D=5$ reaches (end even exceeds) the values observed at the wind turbine position, a maximum velocity deficit of all tests is observed at $x / D=1$ downstream of the large hill. Here, the flow is retarded both due to the large hill as well as due to the wind turbine nacelle as the velocity deficit is $66 \%$ with respect to the undisturbed, free stream flow. Due to flow channeling, the velocity deficit at the wind turbine position immediately downstream of the hill is the smallest in the case of the hill with a bay $(26 \%)$. On the other hand, this is the only case studied where the velocity deficit did not recover until reaching $x / D=5$ as it remains lower $(36 \%)$ than immediately behind the hill $(26 \%)$, likely due to more complex orographic wave breaking phenomena as compared to the hills with a height uniform in the lateral direction. These trends are expected to be even more pronounced in the case of rotating wind turbine blades, e.g. Ainslie [44].

At the wind turbine hub height, velocities remain nearly constant in the lateral direction, as reported in Fig. 7. The results are reported at $z / z_{\text {hub }}=1$ in one lateral direction only $(y / R$ values are always positive) as the preliminary tests indicated that this profile is symmetrical on both sides in the lateral direction from the wind turbine tower. 
a) Wake at $x / D=1$

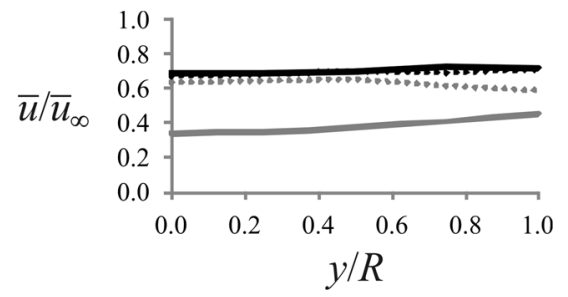

b) Wake at $x / D=3$

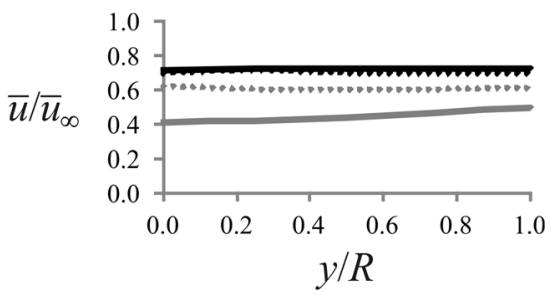

c) Wake at $x / D=5$

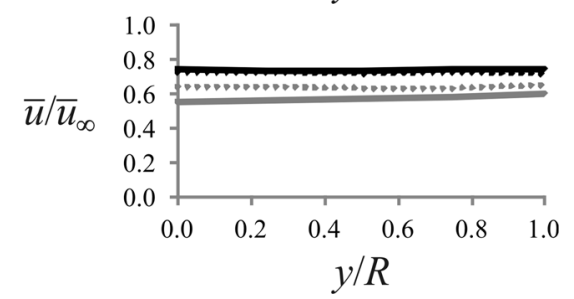

Fig. 7 a) - c) Lateral $(y)$ profiles of the longitudinal $(u)$ flow velocity at different streamwise positions downstream of the wind turbine (wind turbine in place) for different terrain types: black solid line is flat terrain (without a hill), black dotted line is small hill, solid grey line is large hill, dotted grey line is hill with a bay; $x / D$ is nondimensionalized downstream distance of the wind turbine, $D$ is the wind turbine rotor diameter, $\bar{u}_{\infty}$ and $z_{\text {hub }}$ are mean flow velocity in the undisturbed, free stream flow and the wind turbine hub height, respectively.

As for the vertical profiles of the mean velocity in the longitudinal direction $(u)$, the velocity deficit of the mean $u$ velocity in the lateral profiles at all $x / D$ positions is the largest in the case of the large hill and decreasing when moving further downstream of the hill. Namely, the observed velocity deficits for different terrains decrease when moving further downstream of the hill, eventually becoming the same at a sufficient distance from the hill. Furthermore, the wind loading component with respect to the mean flow velocity is expected to remain nearly the same in the lateral direction along the wind turbine rotor blade due to nearly constant mean flow velocities.

Hu et al. [6] and Churchfield et al. [45] indicated the importance of taking the dynamic structural loads induced by atmospheric turbulence into account when designing wind turbines in order to extend their lifetime with respect to structural fatigue. While historically the time-averaged wind loads were used to calculate the mechanical design of wind turbines, the effects of the atmospheric turbulence and respective dynamic wind loads have been receiving an increasing attention in recent years. In particular, the instantaneous wind loads acting on a wind turbine operating in a turbulent atmospheric boundary layer wind could be two to three times greater in comparison to the time-averaged values, Hu et al. [6]. Hence, a particular focus of this study is on integral atmospheric turbulence parameters, i.e. the turbulence intensity in all three directions and the dominant $u$ 'w' turbulent Reynolds stress component as well as their vertical and lateral profiles reported in Figs. 8 to 11. 


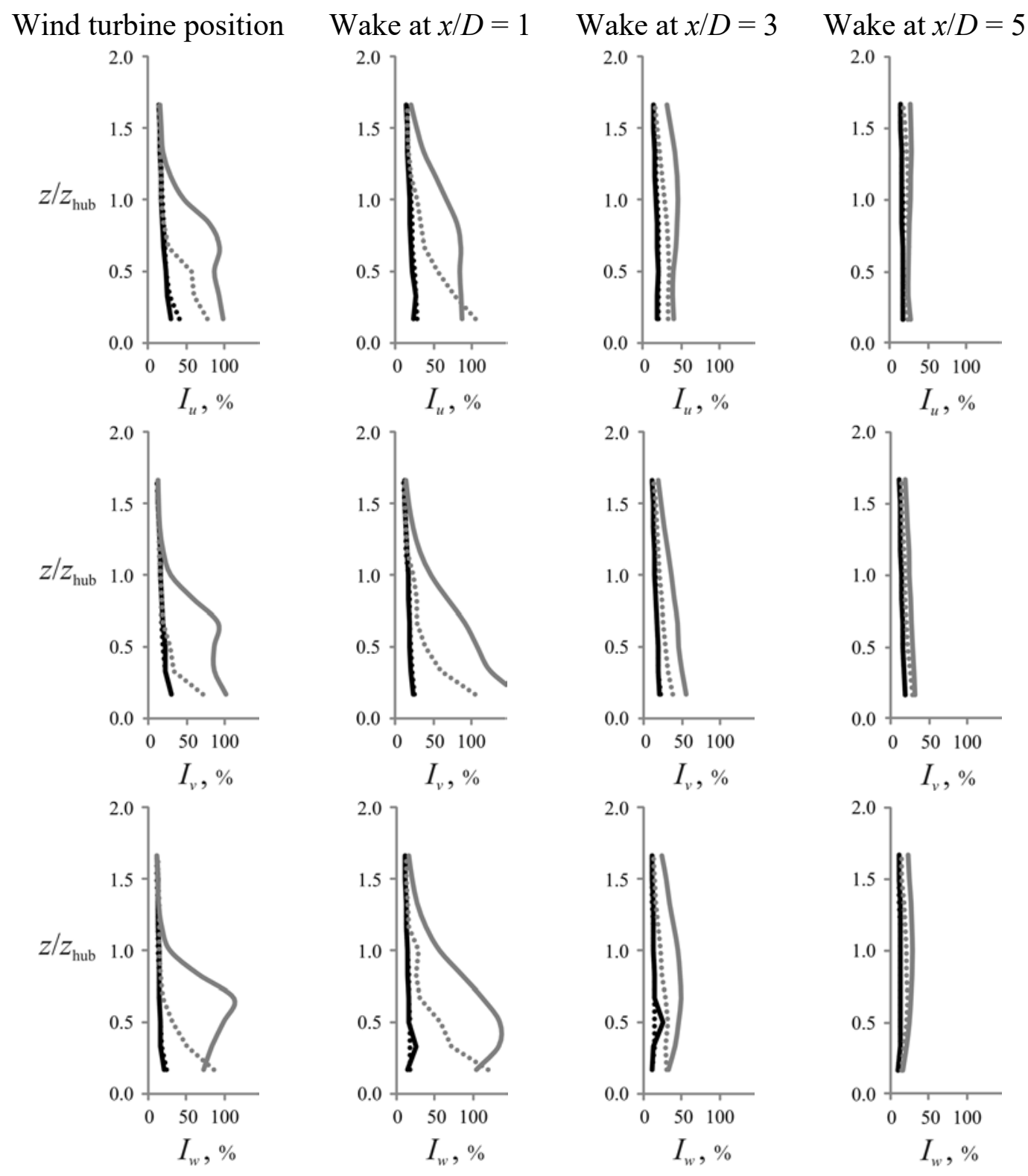

Turbulence intensity $I_{u}$ at the hub height, \%

\begin{tabular}{ccccc} 
& FT & SH & LH & BAY \\
\hline Wind turbine position & 17 & 18 & 47 & 18 \\
Wake at $x / D=1$ & 18 & 21 & 67 & 29 \\
Wake at $x / D=3$ & 17 & 19 & 45 & 28 \\
Wake at $x / D=5$ & 16 & 17 & 27 & 24
\end{tabular}

Fig. 8 Vertical profiles of the turbulence intensity at the position of the wind turbine (without wind turbine in place) as well as at different streamwise positions downstream of the wind turbine (wind turbine in place) for different terrain types: black solid line is flat terrain (without a hill), black dotted line is small hill, solid grey line is large hill, dotted grey line is hill with a bay; all turbulence intensity values normalized locally with a respective mean flow velocity in the main $(x)$ flow direction at the measurement point height; turbulence intensity $I_{u}$ at the hub height is tabulated below diagrams.

Clear trends in vertical profiles of turbulence intensity in all three directions can be seen in Fig. 8. The magnitude of turbulence intensity is generally observed to be considerably greater than commonly recorded in the $\mathrm{ABL}$, which is due to a strong flow pulsation and recirculation downstream of the hill and the wind turbine along with a strong reduction in the 
mean flow velocity. Longitudinal and lateral turbulence intensity profiles are particularly important to be considered with respect to tower vibrations, while the vertical turbulence intensity can influence rotor blades and their unsteady aerodynamics during rotation.

Immediately downstream of the hill at the wind turbine position, the large hill induces the highest turbulence intensity followed by the hill with a bay, while the small hill exhibits low turbulence intensity similar to the flat terrain. Those differences are considerable in the lower part of the wind turbine up to approximately $0.7 z_{\text {hub }}$ as they die out with a further increase in the height, and the turbulence intensity above the hub height is nearly the same for all hill types. The trends observed are similar in all turbulence intensity components $I_{u}, I_{v}$, and $I_{w}$. Along with moving further downstream of the hill, turbulence intensity in all three directions decreases, which trend is particularly pronounced in the case of the large hill and the hill with a bay, and between $x / D=3$ and 5 . It is interesting to observe that at $x / D=5$ the vertical profiles of the turbulence intensity did not start to recover their shape yet in order to regain profile features similar to the $\mathrm{ABL}$ upstream of the hill. In particular, in the $\mathrm{ABL}$ simulation upstream of the hill there is a strong decrease in turbulence intensity with an increase in the height, while at $x / D=5$ in the wind turbine wake, the turbulence intensity remains fairly constant as the height increases. This means that the effect of a hill on atmospheric turbulence is still considerable at that position. In general, the hill can be observed to cause stronger disturbance of turbulence intensity rather than of flow velocity. Due to a stronger turbulence close to the ground surface, the structural fatigue of wind turbine towers is expected to be greater closer to the hill as well as in the case of larger hills.

Lateral profiles of the turbulence intensity in all three directions confirm an increase in turbulence with an increase in the hill height, which is particularly pronounced closer to the hill at $x / D=1$, Fig. 9 .
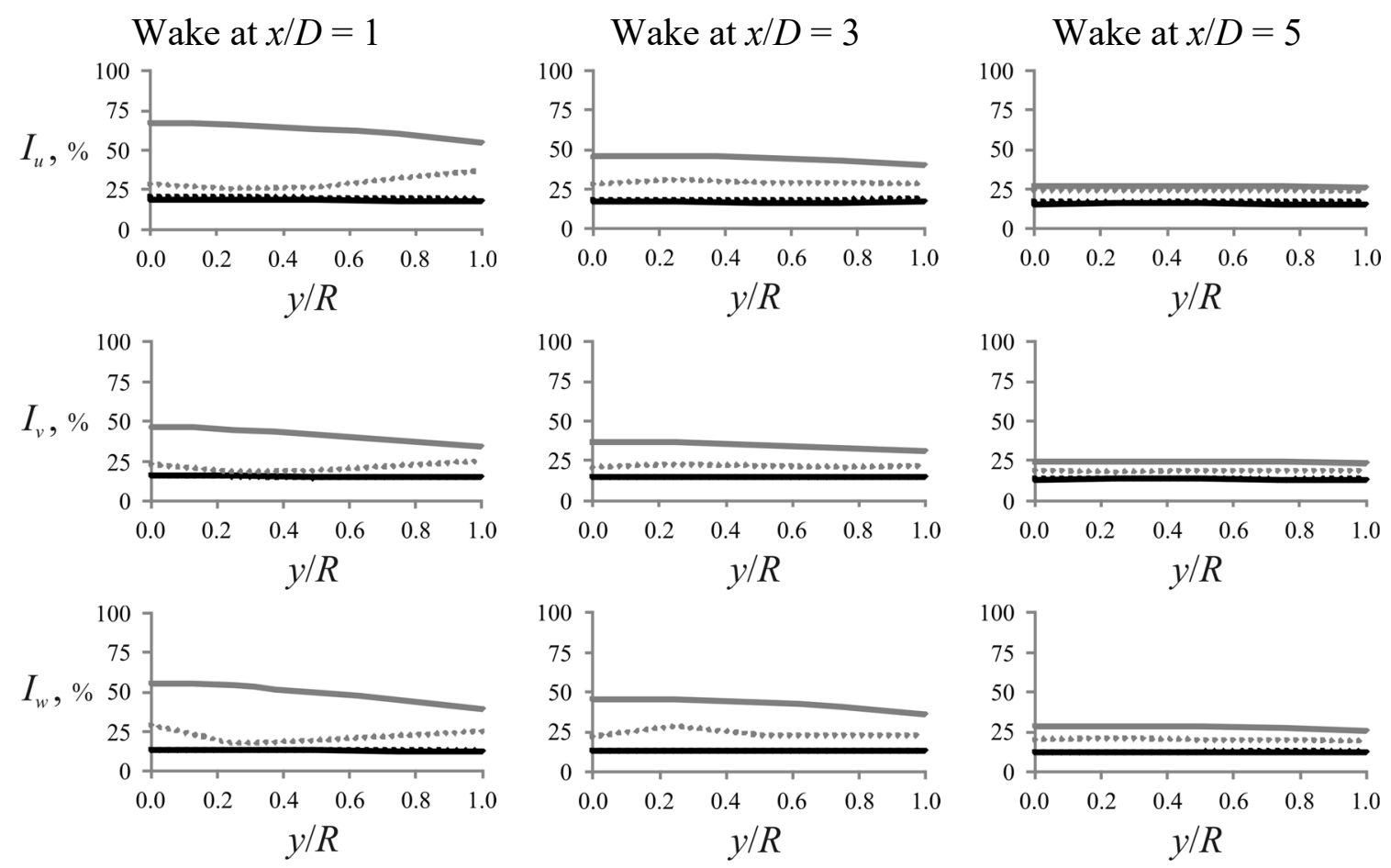

Fig. 9 Lateral profiles of the turbulence intensity at the position of the wind turbine (without wind turbine in place) as well as at different streamwise positions downstream of the wind turbine (wind turbine in place) for different terrain types: black solid line is flat terrain (without a hill), black dotted line is small hill, solid grey line is large hill, dotted grey line is hill with a bay; all turbulence intensity values normalized locally with a respective mean flow velocity in the main $(x)$ flow direction at the measurement point height. 
Further downstream the effects of the large hill and the hill with a bay on the lateral turbulence intensity diminish and these differences are minimal at $x / D=5$. An interesting feature is the constant turbulence intensity in the lateral direction for both the flat terrain and the small hill configurations independently from the distance from the hill. In the case of the large hill the turbulence is at its peak close to the wind turbine nacelle, while the hill with a bay shows a unique trend of slightly increasing the turbulence intensity when the profile approaches the blade tip position at $x / D=1$. The rotor blades are generally expected to receive the same amount of turbulence intensity along the rotor in the lateral direction, particularly in the case of smaller hills and the flat terrain. As large hills cause strong disturbances to the flow, this will create nonuniformities in the lateral profiles of the turbulence intensity when closer to the hill, Fig. 9.

Another integral turbulence parameter considered in this study is the turbulent Reynolds shear stress, i.e. its dominant $u$ 'w' component, as presented in Figs. 10 and 11. While perhaps not that important for wind turbine tower loads, the Reynolds shear stress is considered to be a significant issue when analyzing unsteady wind loading of rotor blades.
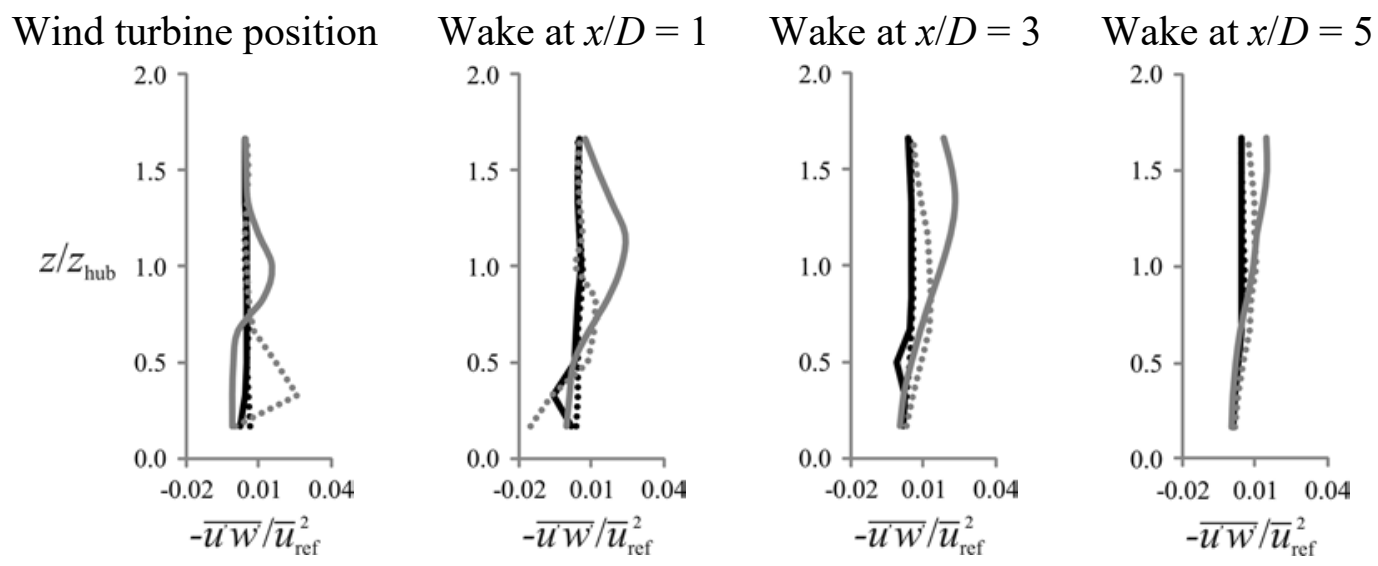

Fig. 10 Vertical profiles of the Reynolds shear stress (normalized using the undisturbed free stream velocity) at the position of the wind turbine (without wind turbine in place) as well as at different streamwise positions downstream of the wind turbine (wind turbine in place) for different terrain types: black solid line is flat terrain (without a hill), black dotted line is small hill, solid grey line is large hill, dotted grey line is hill with a bay.
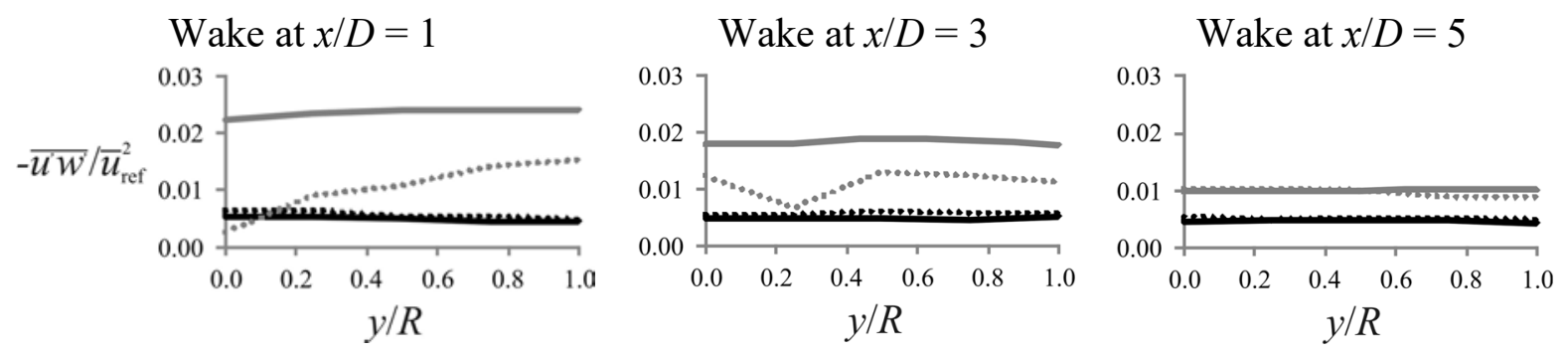

Fig. 11 Lateral profiles of the Reynolds shear stress (normalized using the undisturbed free stream velocity) at the position of the wind turbine (without wind turbine in place) as well as at different streamwise positions downstream of the wind turbine (wind turbine in place) for different terrain types: black solid line is flat terrain (without a hill), black dotted line is small hill, solid grey line is large hill, dotted grey line is hill with a bay.

The Reynolds stress remains nearly constant in the vertical direction in the case of the flat terrain and the small hill. On the other hand, in the large hill scenario strong gradients can be observed in the upper part of the vertical profile when close to the hill (i.e. from the wind turbine position to $x / D=3$ ), which is due to a free shear layer that separates from the hill ridge. In the case of the hill with a bay, strong gradients appear closer to the ground surface 
due to the complex aerodynamics developing because of the flow through the hill pass. Further downstream of the hill (at $x / D=5)$, the effects of the complex terrain diminish as the vertical profiles remain nearly uniform with the height increasing in the case of all terrain types. In the lateral direction, the Reynolds stress remains nearly constant with nearly the same values at all downstream distances in the case of the flat terrain and the small hill. In the case of the large hill, the laterally uniform Reynolds stress is larger in comparison to other terrains, while it decreases when moving further away from the hill, indicating a dominant effect of the hill height on the Reynolds stress.

Strong gradients are observed close to the wind turbine nacelle in the case of the hill with a bay model. This is due to a particularly complex aerodynamics in this configuration. Therefore, the rotor blades are expected to experience greater unsteady wind loads when the wind turbine is placed close to large hills of a complex topography than in the case of smaller hills of a uniform height. In order to mitigate such an adverse effect of a hill on wind loading of rotor blades it is necessary to place the wind turbine further away from the hill as the Reynolds shear stress strongly decreases further downstream of the hill. For example, in the large hill configuration the Reynolds stress at the hub height at the distance $x / D=5$ is less than a half of the Reynolds stress recorded at the wind turbine position immediately downstream of the hill along with negligible gradients in both the vertical and the lateral direction.

\subsection{Spectral analysis of the wind turbine wake}

While the integral turbulence parameters provide valuable information when calculating unsteady aerodynamic loads on wind turbines, it is particularly important to learn about the frequency content of the flow pulsations, also for the purpose of identifying the dominant frequencies of the atmospheric wind. In general, the spectrum of wind pulsations is considered more important than the peak loading in the context of wind-induced dynamic loading of engineering infrastructure (Barnard [46]) as the ultimate goal (particularly) when designing slender engineering structures is to avoid the overlapping of natural frequencies of the structure with dominant frequencies of the wind. Therefore, the power spectral density of longitudinal velocity fluctuations at the position of the wind turbine (without a wind turbine in place) was analyzed for different hills as well as the modifications to the power spectra at the hub height at different positions downstream of the hill, as reported in Figs. 12 and 13, respectively. In Fig. 12, the power spectra of flow velocity fluctuations are observed immediately downstream of the hill along the wind turbine tower height and are used to analyze which parts of the tower will be particularly sensitive to changes in the topography. As the atmospheric turbulence generally deteriorates the wind turbine energy yield, the wind spectral content at the hub height at different distances downstream of the hill is reported in Fig. 13. 
a)

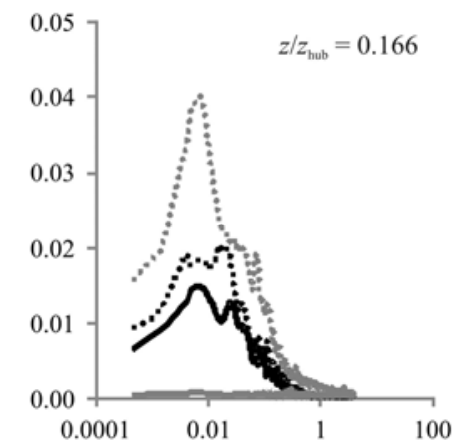

b)

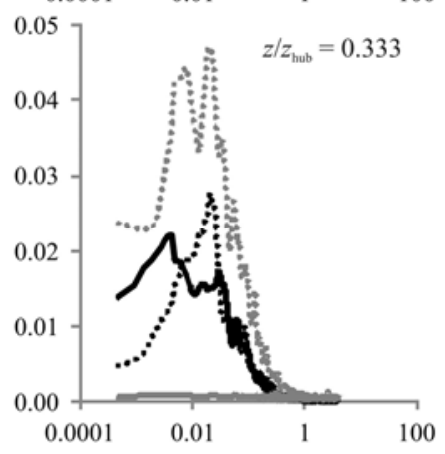

c)

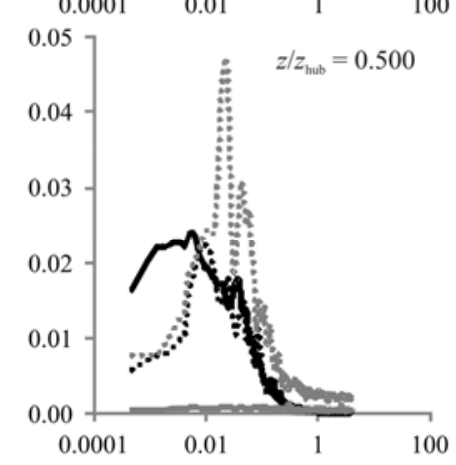

d)

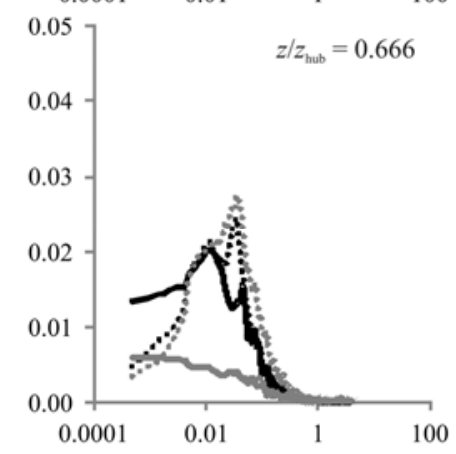

e)

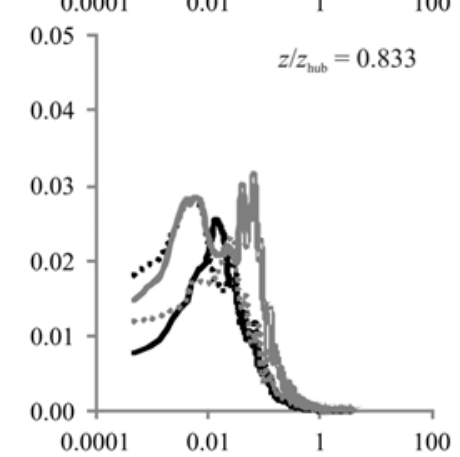

f)

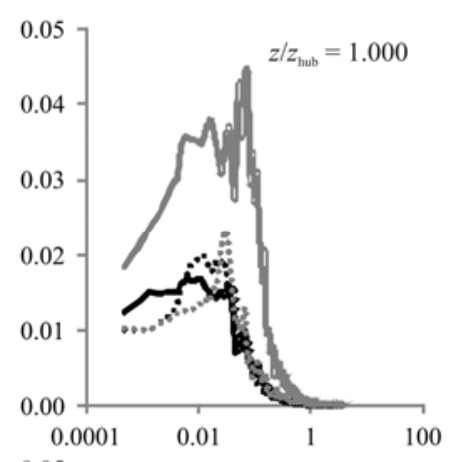

g)

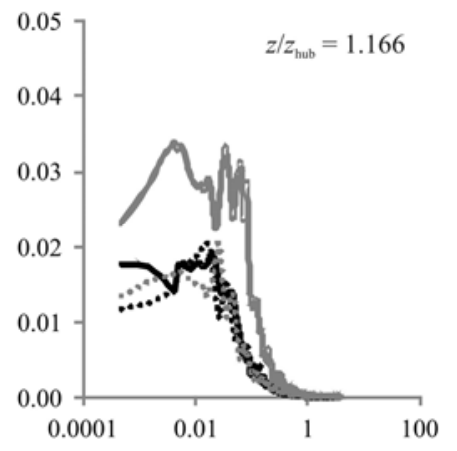

h)
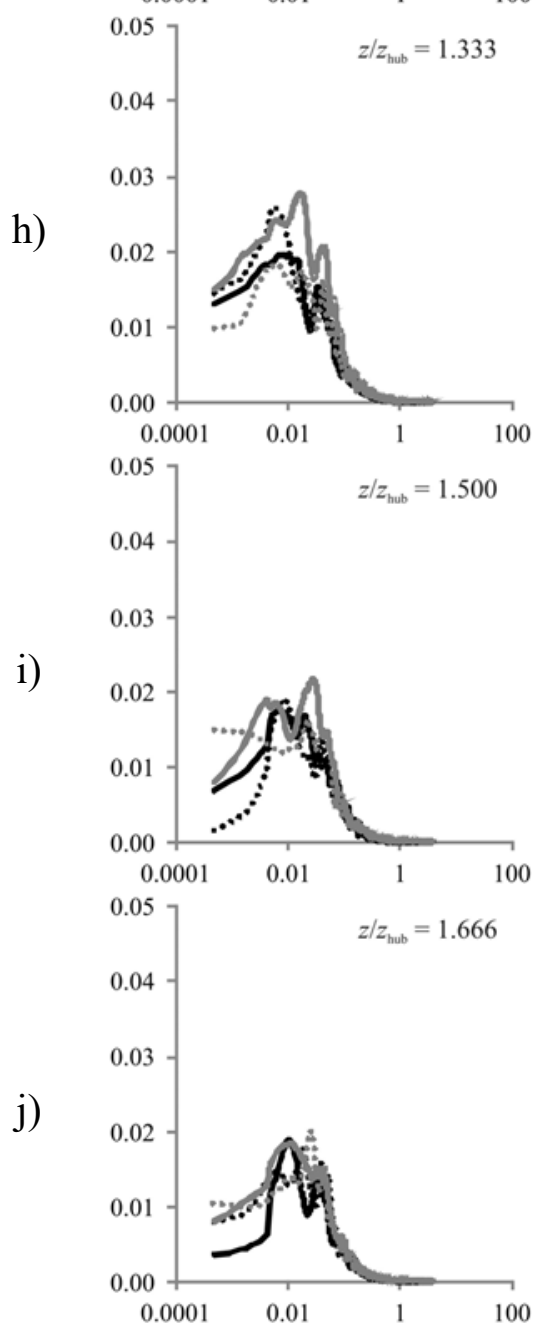

Fig. 12 Power spectral density of longitudinal velocity fluctuations at the position of the wind turbine (without wind turbine in place) for different terrain types: black solid line is flat terrain (without a hill), black dotted line is small hill, solid grey line is large hill, dotted grey line is hill with a bay; $x$-axis is nondimensionalized (reduced) frequency $f \cdot z_{\text {hub }} / \bar{u}_{\infty}, y$-axis is $S_{u}(f)$ in $\mathrm{m}^{2} / \mathrm{s}, f$ is frequency in $\mathrm{Hz}, z_{\text {hub }}$ is wind turbine hub height, $\bar{u}_{\infty}$ is free stream velocity. 
a)

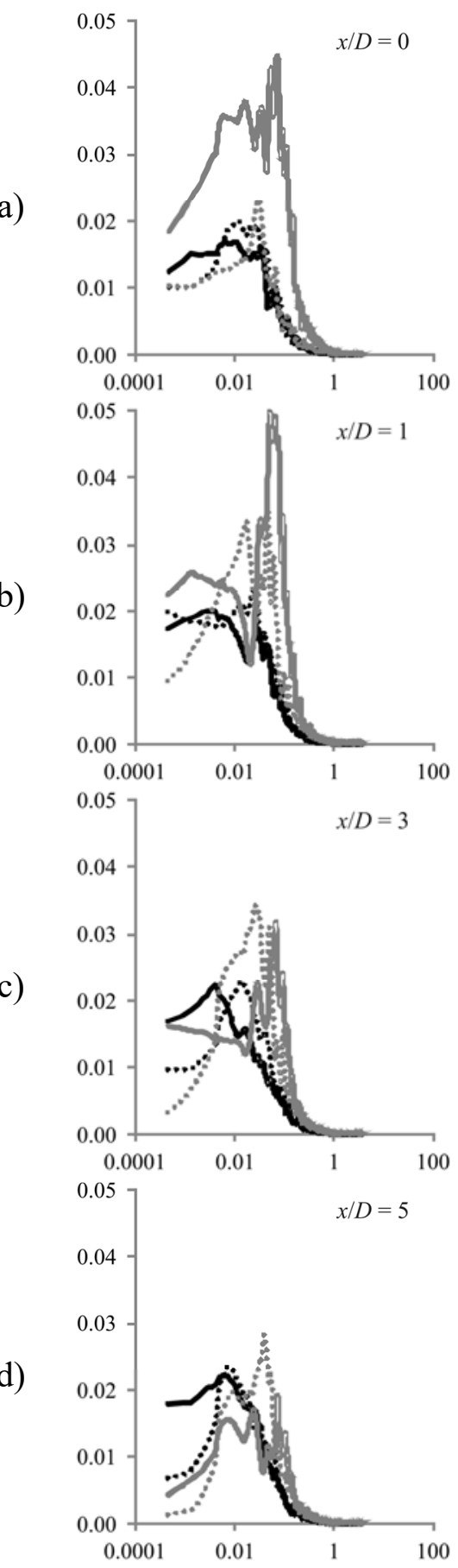

Fig. 13 Power spectral density of longitudinal velocity fluctuations at the hub height at different streamwise positions downstream of the wind turbine (wind turbine in place) for different terrain types: black solid line is flat terrain (without a hill), black dotted line is small hill, solid grey line is large hill, dotted grey line is hill with a bay; $x$-axis is nondimensionalized (reduced) frequency $f \cdot z_{\text {hub }} / \bar{u}_{\infty}, y$-axis is $S_{u}(f)$ in $\mathrm{m}^{2} / \mathrm{s}, f$ is frequency in $\mathrm{Hz}$, $z_{\text {hub }}$ is wind turbine hub height, $\bar{u}_{\infty}$ is free stream velocity.

The peak values in the reported power spectra due to the periodic vortex shedding from the hill are in good agreement with previous atmospheric measurements, wind tunnel experiments and computational modelling, e.g. Trischka [47], Atkinson [48], Schär and Smith [49]. In particular, in the uniform, not stratified flow in the wind tunnel for simple cones and cylinder-cone equivalents Trischka [47] observed Strouhal $(S t=f D / u)$ numbers from 0.23 to 0.29 , where $f$ is the frequency of shedding, $D$ is the characteristic dimension of the obstacle, $u$ is the wind speed. According to the review by Atkinson [48], the Strouhal number can range 
from 0.15 to 0.32 , where the characteristic length scale is the island diameter, while in the computational simulations by Schär and Smith [49] the respective Strouhal number is 0.18. As a representative result of the present study, the peak value of the large hill configuration reported in Fig. 12f corresponds to $S t=f D / u=0.28$. $D$ is the average hill diameter $(0.8 \mathrm{~m})$ calculated as an average of the hill length $(0.6 \mathrm{~m})$ and width $(1 \mathrm{~m}), u$ is the free stream velocity $(15 \mathrm{~m} / \mathrm{s})$, and $f$ is the vortex shedding frequency recorded in this test $(5.25 \mathrm{~Hz})$. In addition, while the present study was carried out for the neutrally stratified ABL, it was previously observed that the thermal stratification of the atmosphere can significantly alter vortex shedding from the hill [47] as well as the flow and turbulence near wind turbines, Chamorro and Porté-Agel [7], Zhang et al. [22]. This indicates that further work is still required with respect to wake characteristics of wind turbines with rotating blades in the thermally stratified ABL developing above a complex terrain.

In the lower part of the wind turbine tower (up to $z / z_{\text {hub }}=0.500$ ), the large hill almost completely shadows the tower as the flow pulsation energy is close to zero at all frequencies. In this height range, the peak is particularly pronounced in the hill with a bay configuration due to the flow through the hill pass generating shed vortices. In the flat terrain configuration, the velocity power spectra look very much like the standard von Kármán [50] design curve, which trend does not significantly change all the way up to the top tip height. In the lower half of the tower height, the small hill induces the velocity power spectra similar to those in the flat terrain configuration. Between the hub height and the top tip height, several interesting turbulence features can be observed. In particular, the turbulent kinetic energy behind the large hill starts to increase at $z / z_{\text {hub }}=0.666$ without a clear peak value observed at this height. However, with a further increase in the height, the peak associated with the vortex shedding from the large hill gains on its strength reaching the maximum at $z / z_{\text {hub }}=1.000$ due to a free shear layer that separates from the hill ridge. With a further increase in the height, this peak weakens, and it is approximately of the same amplitude as in the case of other hills at the top tip height $\left(z / z_{\text {hub }}=1.666\right)$. The peak values and frequencies in the flat terrain, small hill and the hill with a bay configurations are nearly the same above the hub height, indicating that the dominant effects of these terrains are mostly constrained within the range between the ground surface and the hub height.

At the hub height (Fig. 13) immediately downstream of the hill, the flow pulsations are dominant in the large hill configuration, while the power spectra in the flat terrain, small hill, and the hill with a bay configurations are nearly the same. A similar balancing among the spectra for different terrains is observed at $x / D=1$ along with the turbulent energy increase in the hill with a bay configuration, which continues further in the wake for $x / D=3$ and 5 along with a strong decrease in the large hill peak value. In the far wake at $x / D=5$, the large hill effect almost completely dies out with the peak value lower than in all other terrains. With respect to the energy production of a wind turbine near a hill, the higher yield is expected for smaller hills of simpler topography due to weaker flow pulsations at characteristic frequencies in the flat terrain and the small hill configurations. The effects of hills on the velocity power spectra are particularly pronounced closer to the hill up to $x / D=1$ because further downstream $(x / D=3$ and 5$)$ the footprints of the complex terrain on the power spectra are not that strong any more.

\section{Conclusions}

Experiments were designed and carried out in a boundary layer wind tunnel in order to analyze wind characteristics in the wake of a single wind turbine placed downstream of a hilly terrain. The focus was on flow characteristics at the same position downstream of the hill and the wind turbine small-scale models studied on various sizes and shapes of the hill model. 
The wind tunnel simulation of the atmospheric boundary layer (ABL) developing above the flat urban-type terrain was created by using a castellated barrier wall, vortex generators and surface roughness elements. The created ABL simulation is in good agreement with the full-scale conditions in the simulation length scale 1:300. This is confirmed through comparison of wind tunnel and full-scale values of the mean velocity in the main flow direction, the turbulence intensity in the longitudinal, lateral and vertical directions, the dominant $\left(u^{\prime} w^{\prime}\right)$ component of the turbulent Reynolds shear stress, the longitudinal integral length scales of turbulence, and the power spectral density of longitudinal velocity fluctuations.

The hill models were separately exposed to the ABL simulation and the wake characteristics of an alone-standing wind turbine model were studied by using three different hill models (a small and a large hill with laterally uniform height, a hill with a bay). The flat terrain configuration was used as a reference case. The wind turbine model was in the parking position in order to study wind characteristics of a strong wind situation when there is no rotation of rotor blades. Mean flow velocity, turbulence intensity, Reynolds shear stress, and velocity power spectra were studied to gain more understanding about the complex wake physics.

The experimental results indicate a velocity decrease and stronger turbulence in the wind turbine wake in the presence of a hill. In particular, the velocity at the hub height downstream of a hill decreases considerably in comparison to the undisturbed, free stream velocity. The large hill shadows the wind turbine to the largest extent which results in the largest velocity deficit with respect to other terrains. This shadowing effect weakens further downstream of the hill as the differences in the velocities observed in different terrains are fairly small in the far wake. Strong velocity gradients observed around the hub height in the large hill configuration (when the wind turbine is placed immediately downstream of the hill) may create large bending moments due to strong winds acting on the higher part of the wind turbine along with different vortex shedding characteristics of different parts of the structure resulting in deteriorated structural stability. Due to flow channeling, the velocity deficit at the wind turbine position immediately downstream of the hill is the smallest in the hill with a bay configuration, while this is the only case studied where the velocity deficit does not recover until reaching the far wake.

Immediately downstream of the hill, the large hill induces the highest turbulence intensity followed by the hill with a bay, while the small hill exhibits low turbulence intensity similar to the flat terrain. These differences observed for different terrains are considerable in the lower part of the wind turbine, they die out with a further increase in the height, and the turbulence intensity higher than the hub height is nearly the same in all hill configurations. Along with moving further downstream of the hill, the turbulence intensity decreases, which trend is particularly pronounced in the large hill and the hill with a bay configuration. Due to a stronger turbulence observed close to the ground surface, the structural fatigue of wind turbine towers is expected to be greater closer to the hill as well as in the case of larger hills.

The Reynolds stress remains nearly constant in the vertical direction in the flat terrain and the small hill configuration. On the other hand, in the large hill scenario strong gradients can be observed in the upper part of the vertical profile (when close to the hill) due to a free shear layer that separates from the hill ridge. In the hill with a bay configuration, strong gradients appear closer to the ground surface due to a complex aerodynamics developing as a consequence of the flow through the hill pass. Further downstream of the hill, the effects of the complex terrain diminish as the vertical profiles remain nearly uniform in all terrains as the height increases. 
The peak values in the velocity power spectra due to the vortex shedding from the hill ridge are in good agreement with the atmospheric measurements, wind tunnel experiments and computational models. In the lower part of the wind turbine tower, the large hill shadows the tower as the flow pulsation energy is close to zero at all frequencies. In this height range, the peak is particularly pronounced in the hill with a bay configuration due to the flow through the hill pass generating shed vortices. In the flat terrain configuration, the velocity power spectra look very much like the standard von Kármán design curve observed upstream of the complex terrain, which trend does not significantly change all the way up to the top tip height. In the lower half of the tower, the small hill induces the velocity power spectra similar to those in the flat terrain configuration. The peak values and frequencies in the flat terrain, the small hill and the hill with a bay configurations are nearly the same above the hub height, thus indicating the dominant effects of these terrains are mostly constrained within the range between the ground surface and the hub height. Further work is still required to fully elucidate wake characteristics of wind turbines with rotating blades in the thermally stratified ABL developing above a complex terrain.

\section{ACKNOWLEDGEMENTS}

The authors wish to acknowledge the FP7-Marinet and the Croatian Science Foundation IP2016-06-2017 (WESLO) funding. We thank Radoslav Babić, Milan Šulentić, Tomislav Barb and Professor Nebojša Bojčetić as well as the Faculty of Mechanical Engineering and Naval Architecture, University of Zagreb for their support concerning the manufacturing of the experimental models. Helpful discussions with Drs. Claudio Mannini and Enzo Marino are also greatly appreciated.

\section{REFERENCES}

[1] Crespo A, Hernandez J, Frandsen S (1999) Survey of modelling methods for wind turbine wakes and wind farms. Wind Energy 2, 1-24.

https://doi.org/10.1002/(sici)1099-1824(199901/03)2:1<1::aid-we16>3.3.co;2-z

[2] Whale J, Papadopoulos KH, Anderson CG, Helmis CG, Skyner DJ (1996) A study of the near wake structure of a wind turbine comparing measurements from laboratory and full-scale experiments. Solar Energy 56, 621-633. https://doi.org/10.1016/0038-092x(96)00019-9

[3] Kozmar H, Allori D, Bartoli G, Borri C (2018) Wind characteristics in wind farms situated on a hilly terrain. Journal of Wind Engineering and Industrial Aerodynamics 174, 404-410. https://doi.org/10.1016/j.jweia.2018.01.008

[4] Vermeer LJ, Sørensen JN, Crespo A (2003) Wind turbine wake aerodynamics. Progress in Aerospace Sciences 39, 467-510. https://doi.org/10.1016/s0376-0421(03)00078-2

[5] Zhang W, Markfort CD, Porté-Agel F (2012) Near-wake flow structure downwind of a wind turbine in a turbulent boundary layer. Experiments in Fluids 52, 1219-1235. https://doi.org/10.1007/s00348-011-1250-8

[6] Hu H, Yang Z, Sarkar P (2012) Dynamic wind loads and wake characteristics of a wind turbine model in an atmospheric boundary layer wind. Experiments in Fluids 52, 1277-1294. https://doi.org/10.1007/s00348-011-1253-5

[7] Chamorro LP, Porté-Agel F (2010) Effects of Thermal Stability and Incoming Boundary-Layer Flow Characteristics on Wind-Turbine Wakes: A Wind-Tunnel Study. Boundary-Layer Meteorology 136, 515 533. https://doi.org/10.1007/s10546-010-9512-1

[8] Massouh F, Dobrev I (2007) Exploration of the vortex wake behind of wind turbine rotor. Journal of Physics: Conference Series 75, 012036:1-012036:9. https://doi.org/10.1088/1742-6596/75/1/012036

[9] Alfredsson PH, Dahlberg JA (1979) A preliminary wind tunnel study of windmill wake dispersion in various flow conditions. Technical note AU-1499, part 7, FFA, Stockholm, Sweden.

[10] Ebert PR, Wood DH (1997) The near wake of a model horizontal-axis wind turbine - 1. Experimental arrangements and initial results. Renewable Energy 12, 225-243.

https://doi.org/10.1016/s0960-1481(97)00046-3 
[11] Grant I, Parkin PA (2000) DPIV study of the trailing vortex elements from the blades of a horizontal axis wind turbine in yaw. Experiments in Fluids 28, 368-376. https://doi.org/10.1007/s003480050396

[12] Whale J, Anderson CG, Bareiss R, Wagner S (2000) An experimental and numerical study of the vortex structure in the wake of a wind turbine. Journal of Wind Engineering and Industrial Aerodynamics 84(1), 1-21. https://doi.org/10.1016/s0167-6105(98)00201-3

[13] Simms D, Schreck S, Hand M, Fingersh LJ (2001) NREL unsteady aerodynamics experiment in the NASA-AMES wind tunnel: a comparison of predictions to measurements. NREL/TP-500-29494. https://doi.org/10.2172/783409

[14] Vermeer LJ (2001) A review of wind turbine wake research at TU-Delft. In: Proceedings of ASME wind energy symposium, ASME, New York, AIAA-2001-0030, 103-113. https://doi.org/10.2514/6.2001-30

[15] Medici D, Alfredsson PH (2006) Measurements on a wind turbine wake: 3D effects and bluff body vortex shedding. Wind Energy 9, 219-236. https://doi.org/10.1002/we.156

[16] Snel H, Schepers JG, Montgomerie B (2007) The MEXICO project (model experiments in controlled conditions): the database and first results of data processing and interpretation. Journal of Physics: Conference Series 75, 012014:1-012014:11. https://doi.org/10.1088/1742-6596/75/1/012014

[17] Dobrev I, Maalouf B, Troldborg N, Massouh F (2008) Investigation of the wind turbine vortex structure. In: 14th International Symposium on Applications of Laser Techniques to Fluid Mechanics, Lisbon, Portugal.

[18] Sørensen JN (2011) Aerodynamic aspects of wind energy conversion. Annu Rev Fluid Mech 43:427-448. https://doi.org/10.1146/annurev-fluid-122109-160801

[19] Chamorro LP, Porté-Agel F (2009) A Wind-Tunnel Investigation of Wind-Turbine Wakes: BoundaryLayer Turbulence Effects. Boundary-Layer Meteorology 132, 129-149. https://doi.org/10.1007/s10546-009-9380-8

[20] Chamorro LP, Porté-Agel F (2011) Turbulent Wind Inside and Above a Wind Farm: A Wind-Tunnel Study. Energies 4, 1916-1936. https://doi.org/10.3390/en4111916

[21] Markfort CD, Zhang W, Porté-Agel F (2012) Turbulent flow and scalar transport through and over aligned and staggered wind farms. Journal of Turbulence 13(33), 1-36. https://doi.org/10.1080/14685248.2012.709635

[22] Zhang W, Markfort CD, Porté-Agel F (2013) Wind-Turbine Wakes in a Convective Boundary Layer: A Wind-Tunnel Study. Boundary-Layer Meteorology 146, 161-179. https://doi.org/10.1007/s10546-012-9751-4

[23] Hancock PE, Pascheke F (2010) Wind tunnel simulations of wind turbine wake interactions in neutral and stratified wind flow. In: 10th EMS Annual Meeting, 10th European Conference on Applications of Meteorology (ECAM) Abstracts, Zurich, Switzerland.

[24] Cal RB, Lebrón J, Castillo L, Kang HS, Meneveau C (2010) Experimental study of the horizontally averaged flow structure in a model wind-turbine array boundary layer. Journal of Renewable and Sustainable Energy 2, 013106:1-013106:25. https://doi.org/10.1063/1.3289735

[25] Hadžić N, Kozmar H, Tomić M (2014) Offshore renewable energy in the Adriatic Sea with respect to the Croatian 2020 energy strategy. Renewable and Sustainable Energy Reviews 40, 597-607. https://doi.org/10.1016/j.rser.2014.07.196

[26] Botta G, Cavaliere M, Viani S, Pospíšil S (1998) Effects of hostile terrains on wind turbine performances and loads: The Acqua Spruzza experience. Journal of Wind Engineering and Industrial Aerodynamics 7476, 419-431. https://doi.org/10.1016/s0167-6105(98)00038-5

[27] Kozmar H, Allori D, Bartoli G, Borri C (2016) Complex terrain effects on wake characteristics of a parked wind turbine. Engineering Structures 110, 363-374.

https://doi.org/10.1016/j.engstruct.2015.11.033

[28] Augusti G, Spinelli P, Borri C, Bartoli G, Giachi M, Giordano S (1995) The C.R.I.A.C.I.V. atmospheric boundary layer wind tunnel. In: Proceedings of the 9th International Conference on Wind Engineering (ICWE), New Delhi, India.

[29] Simiu E, Scanlan RH (1996) Wind Effects on Structures. John Wiley \& Sons, New York, USA.

[30] Dyrbye C, Hansen S (1997) Wind Loads on Structures. John Wiley \& Sons, New York, USA.

[31] Holmes JD (2015) Wind Loading of Structures. CRC Press, Boca Raton, FL, USA.

[32] Mattuella JML, Loredo-Souza AM, Oliveira MGK, Petry AP (2016) Wind tunnel experimental analysis of a complex terrain micrositing. Renewable and Sustainable Energy Reviews 54, 110-119.

https://doi.org/10.1016/j.rser.2015.09.088 
[33] Counihan J (1969) An improved method of simulating an atmospheric boundary layer in a wind tunnel. Atmospheric Environment 3, 197-214. https://doi.org/10.1016/0004-6981(69)90008-0

[34] Lepri P, Kozmar H, Večenaj Ž, Grisogono B (2014) A summertime near-ground velocity profile of the Bora wind. Wind and Structures 19(5), 505-522. https://doi.org/10.12989/was.2014.19.5.505

[35] Lepri P, Večenaj Ž, Kozmar H, Grisogono B (2015) Near-ground turbulence of the Bora wind in summertime. Journal of Wind Engineering and Industrial Aerodynamics 147, 345-357. https://doi.org/10.1016/j.jweia.2015.09.013

[36] Kozmar H (2011) Characteristics of natural wind simulations in the TUM boundary layer wind tunnel. Theoretical and Applied Climatology 106(1-2), 95-104. https://doi.org/10.1007/s00704-011-0417-9

[37] Kozmar H (2011) Truncated vortex generators for part-depth wind-tunnel simulations of the atmospheric boundary layer flow. Journal of Wind Engineering and Industrial Aerodynamics 99, 130-136. https://doi.org/10.1016/j.jweia.2010.11.001

[38] Kozmar H (2011) Wind-tunnel simulations of the suburban ABL and comparison with international standards. Wind and Structures 14(1), 15-34. https://doi.org/10.12989/was.2011.14.1.015

[39] Kozmar H (2012) Improved experimental simulation of wind characteristics around tall buildings. Journal of Aerospace Engineering 25(4), 670-679. https://doi.org/10.1061/(asce)as.1943-5525.0000167

[40] Kozmar H (2012) Physical modeling of complex airflows developing above rural terrains. Environmental Fluid Mechanics 12(3), 209-225. https://doi.org/10.1007/s10652-011-9224-1

[41] Cook NJ (1978) Determination of the model scale factor in wind-tunnel simulations of the adiabatic atmospheric boundary layer. Journal of Wind Engineering and Industrial Aerodynamics 2(4), 311-321. https://doi.org/10.1016/0167-6105(78)90016-8

[42] Plate EJ (1982) Engineering Meteorology. Elsevier, Amsterdam, The Netherlands.

[43] Manwell J, Mcgowan J, Rogers A (2009) Wind Energy Explained: Theory, Design and Application. John Wiley \& Sons, New York, USA. https://doi.org/10.1002/9781119994367

[44] Ainslie JF (1988) Calculating the flowfield in the wake of wind turbines. Journal of Wind Engineering and Industrial Aerodynamics 27, 213-224. https://doi.org/10.1016/0167-6105(88)90037-2

[45] Churchfield MJ, Lee S, Michalakes J, Moriarty PJ (2012) A numerical study of the effects of atmospheric and wake turbulence on wind turbine dynamics. Journal of Turbulence 13(14), 1-32. https://doi.org/10.1080/14685248.2012.668191

[46] Barnard RH (2000) Predicting dynamic wind loading on cantilevered canopy roof structures. Journal of Wind Engineering and Industrial Aerodynamics 85, 47-57. https://doi.org/10.1016/s0167-6105(99)00119-1

[47] Trischka JW (1980) Cone models of mountain peaks associated with atmospheric vortex streets. Tellus 32(4), 365-375. https://doi.org/10.3402/tellusa.v32i4.10591

[48] Atkinson BW (1981) Meso-scale Atmospheric Circulations, Academic Press, London, UK.

[49] Schär C, Smith RB (1993) Shallow-Water Flow past Isolated Topography. Part 2: Transition to Vortex Shedding. Journal of the Atmospheric Sciences 50(10), 1401-1412. https://doi.org/10.1175/1520-0469(1993)050<1401:swfpit $>2.0 . c 0 ; 2$

[50] Von Kármán T (1948) Progress in the statistical theory of turbulence. In: Proceedings of the National Academy of Sciences of the United States of America 34(11), 530-539. https://doi.org/10.1073/pnas.34.11.530

[51] ESDU (1974) Characteristics of atmospheric turbulence near the ground, Part II: Single point data for strong winds (neutral atmosphere). In: Engineering Sciences Data Unit 74031.

Submitted: $\quad 18.02 .2019$

Accepted: $\quad 28.5 .2019$
Hrvoje Kozmar

Faculty of Mechanical Engineering and Naval Architecture, University of Zagreb Ivana Lučića 5, 10000 Zagreb, Croatia

Davide Allori

Gianni Bartoli

Claudio Borri

Department of Civil and Environmental Engineering, University of Florence Via di Santa Marta 3, 50139 Florence, Italy 


\section{APPENDIX}

a)

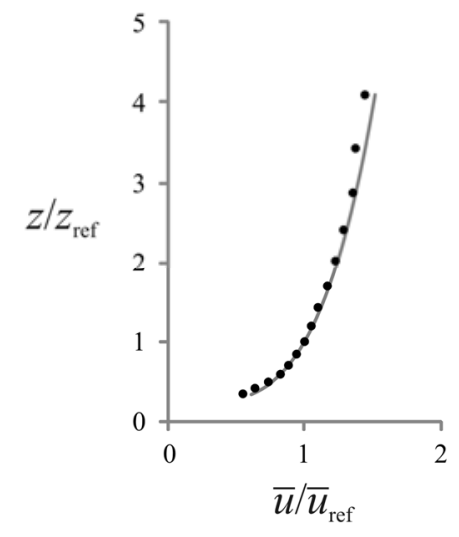

d)

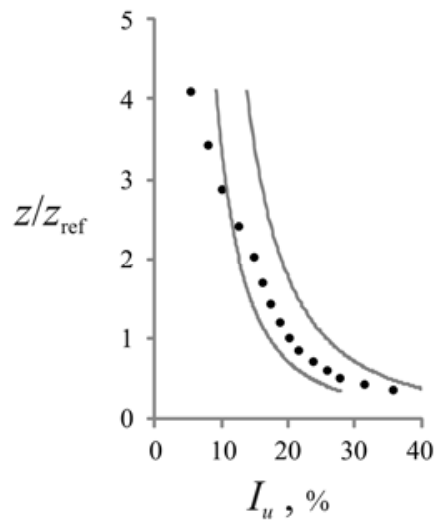

b)

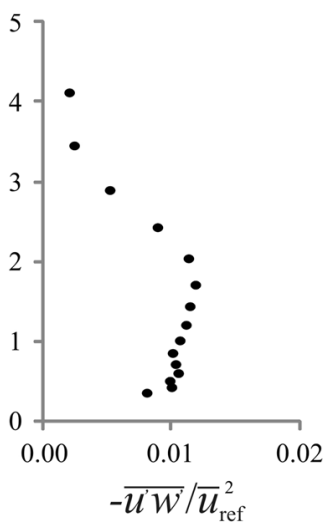

e)

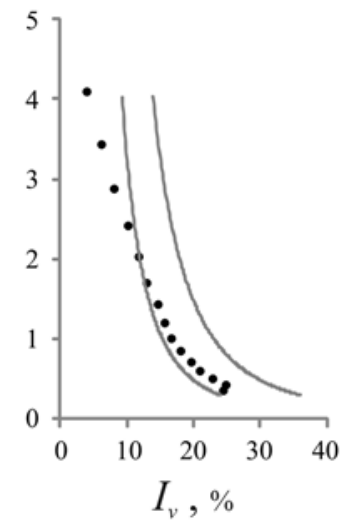

c)

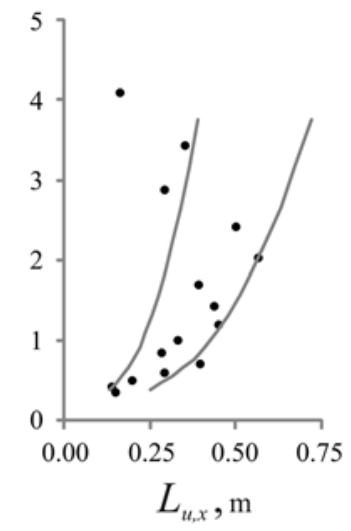

f)

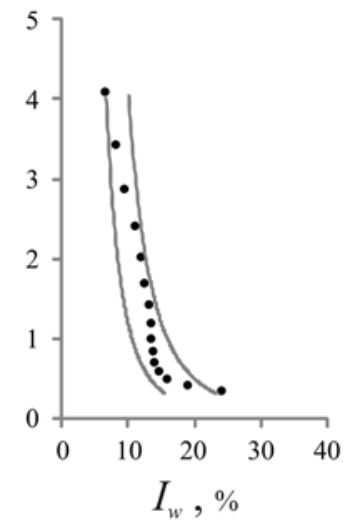

g)

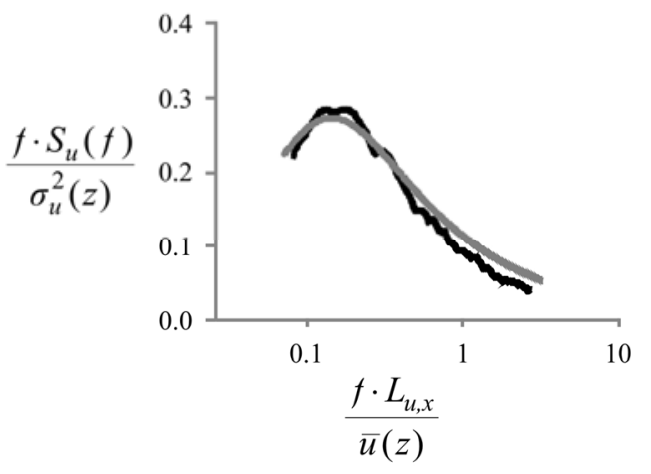

Fig. 14 Characteristic profiles of the created ABL simulation: a) mean flow velocity profile (solid dots are experimental results, solid line is the power law approximation with $\alpha=0.26, d=5 \mathrm{~cm}$ ), b) Reynolds shear stress profile; $\bar{u}_{\text {ref }}=10.2 \mathrm{~m} / \mathrm{s}$ is mean flow velocity in the main flow direction $x$ recorded at the reference height $z_{\text {ref }}=0.216 \mathrm{~m}, \mathrm{c}$ ) longitudinal integral turbulence length scale $L_{u, x}$ (at wind tunnel scale) in comparison with the respective ESDU74031 [51] data scaled-down at the adopted 1:300 simulation length scale with the tolerance $\pm 30 \%$ (solid lines), d) longitudinal turbulence intensity $I_{u}$ profile, e) lateral turbulence intensity $I_{v}$ profile, f) vertical turbulence intensity $I_{w}$ profile; all turbulence intensity values (solid dots) are normalized locally with a mean flow velocity in the main $(x)$ flow direction at the measurement point height, reported in comparison with the respective ESDU74031 [51] data scaled-down at the adopted 1:300 simulation length scale with the tolerance $\pm 20 \%$ (solid lines), g) power spectral density of longitudinal velocity fluctuations (black solid line) at the reference height $z_{\text {ref }}=0.216 \mathrm{~m}$ in comparison with the von Kármán [50] function (grey solid line) 